\title{
ON LADDER HEIGHT DENSITIES AND LAGUERRE SERIES IN THE STUDY OF STOCHASTIC FUNCTIONALS. II. EXPONENTIAL FUNCTIONALS OF BROWNIAN MOTION AND ASIAN OPTION VALUES
}

\author{
MICHAEL SCHRÖDER *
}

\begin{abstract}
In this paper we develop a constructive structure theory for a class of exponential functionals of Brownian motion which includes Asian option values. This is done in two stages of differing natures. As a first step, the functionals are represented as Laguerre reduction series obtained from main results of Schröder (2006), this paper's companion paper. These reduction series are new and given in terms of the negative moments of the integral of geometric Brownian motion, whose structure theory is developed in a second step. Providing a new angle on these processes, this is done by establishing connections with theta functions. Integral representations and computable formulae for the negative moments are thus derived and then shown to furnish highly efficient ways for computing the negative moments. Application of this paper's Laguerre reduction series in numerical examples suggests that one of the most efficient methods for the explicit valuation of Asian options is obtained. The paper also provides mathematical background results referred to in Schröder (2005c).
\end{abstract}

Keywords: Laguerre series methods for stochastic functionals; ladder height density; moment formulae for the integral of geometric Brownian motion; explicit valuation of an Asian option

2000 Mathematics Subject Classification: Primary 60J65; 91B28

Secondary 65C50; 33C45

\section{Introduction}

In this paper we develop an explicit structure theory for exponential functionals of Brownian motion which is constructive and enables their numerical computation. Such functionals are mappings on spaces of functions $f$ that send any such $f$ to the expectation of its image of the value of a process at time $h$. We thus consider functionals of the form

$$
\mathrm{E}\left[f\left(A_{h}^{(v)}\right)\right], \quad \text { where } A_{h}^{(v)}=\int_{0}^{h} \mathrm{e}^{2\left(v u+W_{u}\right)} \mathrm{d} u,
$$

$v$ is any real drift, $W$ is Brownian motion, and the processes $A^{(v)}$ so obtained are integrals of geometric Brownian motion. The motivation for studying these functionals of Brownian motion has its origins in areas as diverse as the physics of random media and mathematical finance; see, for instance, Yor (2001) and the references there. From the perspective of mathematical

Received 23 August 2004; revision received 9 May 2006.

* Postal address: Keplerstrasse 30, D-69469 Weinheim (Bergstrasse), Germany. 
finance, these functionals can be interpreted as values of contingent claims on averages of prices or of rates. As such, they in particular include values of Asian options, a notion reviewed in Section 6.1 which has in fact motivated much of the development in this area; see, for instance, Dufresne (2000), (2005). For these applications in particular, computability of the above functionals becomes a problem which does not seem to be well addressed even today. Therefore, one of the motivations for the present paper is to show how the Laguerre reduction series results of Schröder (2006) can be developed into such methods.

In Schröder (2006), the author focused on two principal ways of applying Laguerre expansions to stochastic functionals. The first goes back to the Asian option valuation context of Dufresne (2000), and the idea is as follows: construct these functionals as probability densities, Laguerre expand the latter, and reconstruct the coefficients of these expansions in terms of the moments of the pertinent process. The idea of the second approach is to expand the densities of the pertinent processes as Laguerre series whose coefficients are then in terms of the moments of those processes, thus obtaining the functionals as integrals of these series, and then to compute these integrals term by term. While in general there are obvious obstructions to both approaches, a framework of sufficient conditions for their validity was developed in Schröder (2006). The first basic idea of the present paper is to put all this to work in the case of stochastic functionals of the integral of geometric Brownian motion.

The first step of our resulting two-step approach is explained in Section 5. Noteworthy here seems, in particular, to be how and from where we obtain the information our framework of sufficient conditions for Laguerre expandability needs: from a study of the asymptotics of the law of the integral of geometric Brownian motion, in which any of the integral representations derived in Yor (1992a), Dufresne (2001), and Schröder (2003) is instrumental. This seems to be a first, but a typical, application of these explicit integral representations, and with hindsight their availability is essential for implementing this part of our approach. This is because, in particular, it is not functionals of the integral of geometric Brownian motion that we find thus amenable to Laguerre expansion but rather those of its reciprocals. Hence, we are led to Laguerre reduction series in the negative moments of the integral of geometric Brownian motion $A^{(v)}$.

While the moments of time-integrated exponential processes such as the integral of geometric Brownian motion are well understood even at the level of Lévy processes, such an understanding is lacking for their negative moments. Developing a structure theory for the negative moments of the integral of geometric Brownian motion may thus qualify as the second principal contribution of this paper, and furnishes the second step of our approach to Laguerre expansion of stochastic functionals of these processes. Referring to Section 7.1 for more details, the idea is as follows. In Dufresne (2000), a functional recurrence rule has been derived for these moments, which we solve. A first type of integral representation for the negative moments of $A^{(v)}$ results, which we interpret in terms of theta functions. This seems to be a novel point of view on $A^{(v)}$ and may qualify as a main contribution of this paper. For the negative moments of $A^{(v)}$, a second type of integral representation is thus obtained, and shown to be representable as series in terms of error functions. Structural considerations suggest the rapid convergence of these series, and they turn out to be most effective tools for numerical computation in Section 8.1.

To illustrate the numerical properties of our two-step approach to Laguerre expansion of stochastic functionals of $A^{(v)}$, we focus on valuing Asian options. This has in fact become an active area of research, pertinent notions of which are recalled in Section 6.1. Referring to Rogers and Shi (1995) for a 'first generation' survey, we here single out four principal approaches that have been developed in recent years. First, using methods from optimal control theory, very 
interesting formulations of the boundary value problem characterizing Asian option prices have been derived by Vecer (2001), (2002) and Vecer and Xu (2004). Second, the most traditional approach to the integral of geometric Brownian motion is based on a spectral analysis of its infinitesimal generator, along the lines of McKean (1956). Eigenfunction integral representations for the law of $A^{(v)}$ have been thus obtained in Monthus and Comtet (1994), Alili (1995), and Comtet and Monthus (1996), and further references can be found in Linetsky (2004). A procedure for approximating Asian option values by series of eigenfunctions was also proposed there, and could provide an interesting perspective. Third, the Hartman-Watson approach of Yor was developed into a constructive method of valuing Asian options in Schröder (2002); main results were summarized in Schröder (2005a). Finally, Schröder (2005c) developed Laguerre reduction approaches alternative to those of Dufresne (2000) and the present paper, but based on this paper's results on negative moments of $A^{(v)}$.

In Section 8, we in fact combine two concepts: first, the Laguerre reduction series for Asian option value functionals, obtained in Section 6 as a consequence of the structure theory of Section 5, and, second, the explicit series for negative moments of $A^{(v)}$, obtained in Section 7 as a consequence of the structure theory there. By replicating the benchmarks obtained using the alternative Hartman-Watson approach in Schröder (2002), (2005a), one of the most efficient numerical methods for explicitly computing the Asian option price functionals thus results. More precisely, we find that computing such prices to accuracies of ten or more decimal places now requires some fifteen to twenty series terms and can be computed in times almost in the millisecond range. This is true even if volatilities or times to maturity are small, situations where difficulties have been noted with all other methods. We would be more than gratified if the present paper furnished more starting points for such work connecting finance and stochastics.

\section{Preliminaries on Laguerre expansions}

\subsection{Laguerre series}

In this section we recall pertinent properties of Laguerre polynomials from Lebedev (1972, Section 4), Andrews et al. (1999, Chapter 6), and Sansone (1991). Fixing any real number $\alpha>$ -1 , for any nonnegative integer $m$ the $m$ th $\alpha$-Laguerre polynomial, $L_{m}^{\alpha}(z)$, is

$$
L_{m}^{\alpha}(z)=\sum_{k=0}^{m} \alpha_{m, k} z^{k}, \quad \text { where } \alpha_{m, k}=\frac{(-1)^{k}}{k !}\left(\begin{array}{l}
m+\alpha \\
m-k
\end{array}\right),
$$

for any complex $z$. Their structural setting is a generalization of the classical Hilbert spaces of square-integrable functions. Let $L_{\alpha}^{2}(0, \infty)$ be the Hilbert space of all complex-valued functions $F$ on the positive reals that are $\alpha$-square integrable, i.e. that satisfy

$$
\|F\|_{\alpha}^{2}=\int_{0}^{\infty} w_{\alpha}(x)|F|^{2}(x) \mathrm{d} x<\infty,
$$

with the weight $w_{\alpha}$ on $(0, \infty)$ given by

$$
w_{\alpha}(x)=x^{\alpha} \mathrm{e}^{-x}
$$

Then, $L_{\alpha}^{2}(0, \infty)$ carries the sesquilinear form $\langle,\rangle_{\alpha}$ given by

$$
\langle F, G\rangle_{\alpha}=\int_{0}^{\infty} w_{\alpha}(x) F(x) \overline{G(x)} \mathrm{d} x,
$$


and has an orthogonal basis furnished by the $L_{m}^{\alpha}$, which satisfy $\left\|L_{m}^{\alpha}\right\|_{\alpha}^{2}=\Gamma(m+\alpha+1) / m !$. By expressing any $F$ in $L_{\alpha}^{2}(0, \infty)$ in this basis, we obtain its $\alpha$-Laguerre series,

$$
F=\sum_{m=0}^{\infty} c_{m} L_{m}^{\alpha}, \quad \text { where } c_{m}=\frac{\left\langle F, L_{m}^{\alpha}\right\rangle_{\alpha}}{\left\langle L_{m}^{\alpha}, L_{m}^{\alpha}\right\rangle_{\alpha}}
$$

for any nonnegative integer $m$. The $c_{m}$ are the $\alpha$-Laguerre coefficients of this series, whose convergence to $F$ is in $\alpha$-mean: $\lim _{M \rightarrow \infty}\left\|F-\sum_{m=0}^{M} c_{m} L_{m}^{\alpha}\right\|_{\alpha}=0$.

\subsection{Growth measures}

In this section we recall the local growth measures, $\delta$ and $\gamma$, of Schröder (2005c, Section 2.3), which encode how Laguerre expandability is determined by local data. They apply to any complex-valued function $f$ on the positive reals, and the idea of them is to describe the behaviour of $f$ at any point $A$ of the extended nonnegative reals, $[0, \infty]$, by comparison with that of power mappings and powers of the exponential function, respectively. The exponential growth order, $\delta_{A}(f)$, of $f$ near $A$ is the supremum over all real $\delta$ such that $\lim _{x \rightarrow A} \mathrm{e}^{\delta x} f(x)=0$ :

$$
\delta_{A}(f)=\sup \left\{\delta \in \mathbb{R}: \lim _{x \rightarrow A} \mathrm{e}^{\delta x} f(x)=0\right\} .
$$

The polynomial growth order, $\gamma_{A}(f)$, of $f$ near $A$ is defined as follows: if $A$ is finite it is the supremum over all real $\gamma$ such that $\lim _{x \rightarrow A} f(x) / x^{\gamma}=0$, i.e.

$$
\gamma_{A}(f)=\sup \left\{\gamma \in \mathbb{R}: \lim _{x \rightarrow A} f(x) / x^{\gamma}=0\right\} ;
$$

otherwise it is defined as $\gamma_{\infty}(f)=\gamma_{0}\left(f^{*}\right)$, where $f^{*}(x)=f(1 / x)$. The respective sets of real numbers $\delta$ and $\gamma$ defining these growth orders may be empty, in which case the respective suprema both equal $-\infty$.

Basic properties of these growth measures are discussed in Schröder (2005c, Section 2.3). In particular, we recall the following Laguerre expansion criterion for a function to have an $\alpha$-Laguerre series: any continuous function $f:(0, \infty) \rightarrow \mathbb{C}$ is in $L_{\alpha}^{2}(0, \infty)$ if it satisfies $2 \gamma_{0}(|f|)>-(\alpha+1)$ and $2 \delta_{\infty}(|f|)>-1$.

\section{Preliminaries on theta functions}

\subsection{Theta functions}

Following Mumford (1983), (1984), (1991), in this section we collect pertinent facts about the classical theta function, $\vartheta$, given, for any complex $z$ and any positive real $w$, by

$$
\vartheta(z \mid w)=\sum_{m \in \mathbb{Z}} \chi_{2(z+m)}(w), \quad \text { where } \chi_{a}(w)=\frac{1}{\sqrt{\pi w}} \exp \left(-\frac{a^{2}}{4 w}\right),
$$

for any complex $a$. This series converges absolutely, and uniformly on compact sets. Thus, $\vartheta$ can be seen as a holomorphic function on the product of the complex plane and the complex upper half-plane. In the theory of modular forms this is usually done such that the above theta function is considered to be evaluated not at $w$ but at the point $\mathrm{i} \pi w$. Theta functions were conceived by Jacobi (1804-1851). Since then they have been developed into a basic class of holomorphic modular forms interrelated with such diverse areas of mathematics as 
number theory, algebraic geometry, and partial differential equations. Two such interrelations, essentially going back to Jacobi, are to be described.

To describe the first, recall that $w^{-1 / 2} \mathrm{e}^{-\pi x^{2} / w}$ is the fundamental solution to the onedimensional heat equation with a delta function at $x=0$ as initial data at $w=0$. Thus, with real arguments, $\vartheta$ can be seen as the superposition of infinitely many such solutions with delta functions at the integers $x=n$ as initial data.

The second interrelation is with modular forms. From this point of view, theta functions can be characterized by a periodicity with respect to each of their two variables. The behaviour with respect to the second variable is deeper and more subtle than that with respect to the first. It is expressed by a functional equation, the Jacobi transformation formula, with respect to the second variable. For real arguments, it asserts the following remarkable identity, for any real numbers $w>0$ and $x$ (see Mumford (1983, p. 33, p. 4)):

$$
1+2 \sum_{m=1}^{\infty} \mathrm{e}^{-w(\pi m)^{2}} \cos (2 \pi m x)=\sum_{m \in \mathbb{Z}} \frac{1}{\sqrt{\pi w}} \exp \left(-\frac{(x+m)^{2}}{w}\right) .
$$

Providing a second series for $\vartheta(x \mid w)$, the left-hand side of this identity is oscillatory and rapidly convergent for large $w$, whereas its right-hand side is positive and rapidly convergent for small $w$. The Jacobi transformation formula is proved by Fourier analysis, exhibiting $\vartheta$ as the Fourier expansion with respect to its second argument. It furnishes the link to the circle of ideas about counting quadratic forms over the integers that Jacobi originally set out to study. For us it will be the property that makes the numerics work.

\subsection{Estimates}

In this section we collect estimates for the remainder terms, $r_{k, M}$, of the two series for $\vartheta$ of Section 3.1 . For any real $x$, they are defined by

$$
\begin{aligned}
& r_{1, M}(x, w)=\sum_{|m| \geq M+1} \chi_{2(x+m)}(w), \\
& r_{2, M}(x, w)=2 \sum_{m \geq M+1} \mathrm{e}^{-(\pi m)^{2} w} \cos (2 \pi m x),
\end{aligned}
$$

for any integer $M \geq 0$ and any real $w>0$. The precise results, making explicit the complementary convergence properties of the series noted in Section 3.1, are as follows.

Proposition 3.1. For any real $x$, we have the estimates

$$
2 r_{1, M}(x, w) \leq \operatorname{Erfc}\left((M+x) / w^{1 / 2}\right)+\operatorname{Erfc}\left((M-x) / w^{1 / 2}\right)
$$

and

$$
\left|r_{2, M}(x, w)\right| \leq(\pi w)^{-1 / 2} \operatorname{Erfc}\left(\pi w^{1 / 2} M\right),
$$

for any integer $M \geq 0$ and any real $w>0$, where $\operatorname{Erfc}(z)=(2 / \sqrt{\pi}) \int_{[z, \infty)} \mathrm{e}^{-u^{2}} \mathrm{~d} u$ is the complementary error function.

Proposition 3.2. If $\sum_{m \in \mathbb{Z}} \theta_{m}$ is either of the two series for $\vartheta(x \mid w)$ of Section 3.1, then

$$
0 \leq \vartheta(x \mid w) \leq \sum_{m \in \mathbb{Z}}\left|\theta_{m}\right| \leq 1+\min \left\{\chi_{0}(w), \chi_{2 x}(w)\right\}
$$

Hence, $\vartheta$ is bounded, in particular, on $\mathbb{R} \times \mathbb{R}_{\geq \varepsilon}$, for any positive real $\varepsilon$. 
The estimates of Proposition 3.1 can be shown using the integral criterion for series convergence. They imply Proposition 3.2 on setting $M=0$. See Schröder (2005c, Section 6.9) for more detail.

\section{Preliminary formulae}

In this section we collect definitions and basic properties of the main functions to be used in the sequel. We denote by $\operatorname{WErfc}(z)=\mathrm{e}^{z^{2}} \operatorname{Erfc}(z)$ the weighted complementary error function, where $\operatorname{Erfc}(z)$ is as defined in Proposition 3.1 for any complex $z$. Let $(\lambda)_{n}$ denote the $n$th Pochhammer symbol of any complex $\lambda$, which is recursively defined by $(\lambda)_{0}=1$ and $(\lambda)_{n+1}=$ $(\lambda+n)(\lambda)_{n}$ for any nonnegative integer $n$.

\subsection{Definitions}

The functions to be considered are defined on the positive reals $h$ and depend on the real parameter $a$. The first two, $A_{a}$ and $B_{a}$, are given by

$$
A_{a}(h)=\sqrt{2 / \pi} h^{-a} \quad \text { and } \quad B_{a}(h)=a \operatorname{WErfc}((a / 2) \sqrt{2 h}) .
$$

Since the latter function computes tails of the normal distribution, $A_{a}$ and $B_{a}$ are directly connected with Brownian motion.

The construction of the remaining two functions mirrors the Jacobi transformation formula for theta functions. For any $B$ in the extended positives, $[0, \infty]$, and any integer $m \geq 1$, they are given by the integrals

$$
C_{a, m}(B, h)=\int_{0}^{B} \chi_{a}(w) Q_{m}(h, w) \mathrm{d} w \quad \text { and } \quad D_{a, m}(B, h)=\int_{B}^{\infty} \mathrm{e}^{-a^{2} w} Q_{m}(h, w) \mathrm{d} w,
$$

where $\chi_{a}(w)=\mathrm{e}^{-a^{2} /(4 w)} / \sqrt{\pi w}$ from Section 3.1 and

$$
Q_{m}(h, w)=\frac{(2 w)^{m-1}}{(2 h w+1)^{m+1 / 2}} .
$$

In the limiting case $B=\infty$, we here let $C_{a, m}(h)=C_{a, m}(\infty, h)$ to simplify notation. Both functions are finite linear combinations of weighted truncated moments of half-integral-degree gamma variables, as we now show.

\subsection{Properties}

The structure of the functions $C_{a, m}$ and $D_{a, m}$ is in fact studied in terms of the functions $G_{n}$ and $G_{n}^{*}$, for any integer $n \geq 1$. For any real $x>0$, these are given by

$$
G_{n}(x)=2 \mathrm{e}^{x} \mathrm{E}\left[\mathbf{1}_{[\sqrt{x}, \infty)}\left(W_{1 / 2}\right) W_{1 / 2}^{-n}\right] \quad \text { and } \quad G_{n}^{*}(x)=x^{n} G_{n}(x)
$$

as weighted truncated moments of Brownian motion $W$ at time $\frac{1}{2}$, and have the following two representations.

Proposition 4.1. For any integer $n \geq 1$ and any real $x>0$, we have

$$
G_{n}(x)=\frac{\mathrm{e}^{x}}{\sqrt{\pi}} \int_{x}^{\infty} \frac{\mathrm{e}^{-w}}{w^{n+1 / 2}} \mathrm{~d} w=\frac{\mathrm{e}^{x}}{\sqrt{\pi}} \Gamma\left(\frac{1}{2}-n, x\right)
$$

and

$$
G_{n}(x)=\frac{(-1)^{n}}{\left(\frac{1}{2}\right)_{n}} \operatorname{WErfc}(\sqrt{x})+\frac{1}{\sqrt{\pi}} \sum_{\ell=0}^{n-1}(-1)^{n-1-\ell} \frac{\left(\frac{1}{2}\right)_{\ell}}{\left(\frac{1}{2}\right)_{n}} \frac{1}{x^{\ell+1 / 2}} .
$$


Here the first representation is in terms of the complementary incomplete gamma function, given by $\Gamma(s, x)=\int_{x}^{\infty} u^{s-1} \mathrm{e}^{-u} \mathrm{~d} u$ for any real $x>0$ and $s$; see Erdélyi et al. (1953, Chapter IX) or Press et al. (1992, Section 6.2). It follows immediately from the definitions. The second representation follows from the first using partial integration: in $\Gamma\left(\frac{1}{2}-n, x\right)=\int_{x}^{\infty} w^{-(n+1 / 2)} \mathrm{e}^{-w} \mathrm{~d} w$ we interpret $w^{-(n+1 / 2)}$ as the $n$th derivative of $w^{-1 / 2}$ according to the relation $\left(w^{-1 / 2}\right)^{(n)}=(-1)^{n}(1 / 2)_{n} w^{-(n+1 / 2)}$, and then use partial integration to successively lower the order from $n$ to 0 . This representation shows how $G_{n}$ is built up by adding power functions to the weighted complementary error function, WErfc.

The functions $G_{n}$ are the building blocks of the functions $C_{a, m}$ and $D_{a, m}$. With the definitions

$$
b_{a}=\frac{a^{2}}{2} \quad \text { and } \quad \beta_{m, k}=(-1)^{k}\left(\begin{array}{c}
m-1 \\
k
\end{array}\right), \quad k \in\{0, \ldots, m-1\},
$$

the following two results make this precise.

Proposition 4.2. We have $C_{a, m}(0, h)=0$ and, letting $\xi_{B}(h)=h+1 /(2 B)$ for $B \neq 0$,

$$
C_{0, m}(B, h)=\frac{\xi_{B}(h)^{1 / 2-m}}{\left(m-\frac{1}{2}\right) \sqrt{2 \pi}}
$$

and, for nonzero $a$,

$$
C_{a, m}(B, h)=\frac{1}{|a|} \frac{b_{a}^{m}}{\mathrm{e}^{b_{a} /(2 B)}} G_{m}\left(b_{a} \xi_{B}(h)\right) .
$$

Proposition 4.3. We have $D_{a, n}(\infty, h)=0$ and, letting $\eta_{B}(h)=1+2 B h$ for finite $B$,

$$
h^{m} D_{0, m}(B, h)=\sum_{k=0}^{m-1} \frac{\beta_{m, k}}{2 k+1} \eta_{B}(h)^{-(k+1 / 2)}
$$

and, for nonzero a,

$$
h^{m} D_{a, m}(B, h)=\frac{\sqrt{\pi}}{2} \sum_{k=0}^{m-1} \beta_{m, k} \frac{\left(b_{a} / h\right)^{k+1 / 2}}{\mathrm{e}^{2 B b_{a}}} G_{k+1}\left(\frac{b_{a}}{h} \eta_{B}(h)\right) .
$$

Proof of Proposition 4.2. The vanishing property for $B=0$ is clear by construction, so assume that $B \neq 0$. In the defining integral for $C_{a, m}(B, h)$, then change variable according to $y(w)=h+1 /(2 w)$, to obtain

$$
C_{a, m}(B, h)=\frac{1}{2 \sqrt{2 \pi}} \int_{y(B)}^{\infty} \mathrm{e}^{-b_{a}(y-h)} \frac{\mathrm{d} y}{y^{m+1 / 2}} .
$$

The formula for $C_{0, m}$ follows by direct integration. For $a \neq 0$, change variable according to $x=b_{a} y$, to obtain

$$
C_{a, m}(B, h)=\frac{1}{\sqrt{2}}\left(b_{a}\right)^{m-1 / 2} \mathrm{e}^{b_{a} h} \int_{L_{B}}^{\infty} \frac{\mathrm{e}^{-x}}{x^{m+1 / 2}} \frac{\mathrm{d} x}{\sqrt{\pi}},
$$

where $L_{B}=b_{a}(h+1 /(2 B))=b_{a} \xi_{B}(h)$. The exponentially weighted integral factor is equal to $\mathrm{e}^{-b_{a} /(2 B)} G_{m}\left(L_{B}\right)$, and the proof of Proposition 4.2 is thus complete. 
Proof of Proposition 4.3. The vanishing property for $B=\infty$ is clear by construction. Thus, assume that $B$ is finite. For $a=0$, computing $D_{a, m}(B, h)$ reduces to integrating $Q_{m}(h, w)=$ $(2 w)^{m-1} /(2 h w+1)^{m+1 / 2}$. Thus, change variable according to $x(w)=2 w h+1$ and binomially expand the numerator, $(x-1)^{m-1}$, of the resulting integrand. The required formula follows on integration of the result,

$$
D_{0, m}(B, h)=\frac{1}{2 h^{m}} \sum_{k=0}^{m-1} \beta_{m, k} \int_{x(B)}^{\infty} \frac{\mathrm{d} x}{x^{k+3 / 2}} .
$$

For $a \neq 0$, change variable according to $x(w)=a^{2}(w+1 /(2 h))$ in the defining integral for $D_{a, m}$ and binomially expand the numerator of the rational factor, $\left(x / a^{2}-1 /(2 h)\right)^{m-1}$, which results, to obtain

$$
D_{a, m}(B, h)=\frac{\mathrm{e}^{a^{2} /(2 h)}}{2 \sqrt{2}} \sum_{k=0}^{m-1} \frac{\beta_{m, k}}{(2 h)^{k}} \int_{x(B)}^{\infty} \mathrm{e}^{-x}\left(\frac{x}{a^{2}}\right)^{m-1-k}\left(\frac{h x}{a^{2}}\right)^{-(m+1 / 2)} \frac{\mathrm{d} x}{a^{2}} .
$$

Collecting terms then completes the proof of Proposition 4.3.

\section{Reduction series for exponential functionals of Brownian motion}

Exemplifying main results of Schröder (2006), in this section we develop Laguerre reduction series for a class of exponential functionals of Brownian motion which includes option values.

\subsection{Basic setting}

This paper focuses on stochastic functionals of two processes: the integral of geometric Brownian motion, $A^{(v)}$, for any real $v$, and the reciprocals of the integral of geometric Brownian motion, $Y_{d}$, for any positive real $d$. They are given by

$$
A_{h}^{(v)}=\int_{0}^{h} \mathrm{e}^{2\left(v u+W_{u}\right)} \mathrm{d} u \quad \text { and } \quad Y_{d, h}=\frac{d}{A_{h}^{(v)}},
$$

for any real $h>0$. Adopting the setting introduced in Schröder (2006, Sections 4.1, 4.2, and 5.1), we then consider expectations

$$
\mathrm{E}[\rho(Y)], \quad \text { where } Y=Y_{d, h} .
$$

Here $\rho$ is any function on the positive reals such that $\rho(Y)$ is $\mathcal{F}_{T}$-integrable and the following holds: we have a factorization

$$
\rho(x)=\psi(x) \varphi(a, x), \quad x \in(0, \infty),
$$

with functions $\psi$, on the positive reals, and $\varphi$, on two copies of the positive reals, such that the triple $(\psi, \varphi, Y)$ is admissible in the sense of Schröder (2006, Definition 4.1). Thus, we moreover assume that $\mathrm{E}\left[\psi(Y) \mid \mathcal{F}_{t}\right]$ is finite and positive, and that there are functions $\chi$ and $L$ from the positive reals into themselves such that, for any real $c>0$, we have $\varphi(c,(0, L(c)])=\{0\}$ and $\varphi(\lambda c, \lambda x)=\chi(\lambda) \varphi(c, x)$ for any reals $\lambda, x>0$. While Schröder (2006, Section 4.3) gave a systematic construction of such functions $\varphi$, immediate examples are furnished by the pay-off functions of call options; see Schröder (2006, Example 4.2) for a discussion.

For convenience, we in addition assume $\psi$ to be a continuous function on the positive reals, with the growth measures of Section 2.2 satisfying $\gamma_{0}(\psi), \delta_{\infty}(\psi)>-\infty$, and for any real $c>0$ denote by $\varphi_{c}$ the function given by $\varphi_{c}(x) \equiv \varphi(c, x)$, for any real $x>0$. 


\subsection{The first type of reduction series}

Developing reduction series is the first principal step of our approach to Laguerre expansion of stochastic functionals. In continuation of Section 5.1, this is exemplified in the present section by making explicit the first of the two types of Laguerre reduction series of Schröder (2006, Section 6) for the above stochastic functionals of $Y_{d, h}$. With the proof postponed to Section 5.5, the precise form of the first of these Laguerre reduction series is as follows.

Theorem 5.1. In the setting of Section 5.1, let $c>0, \alpha>-1$, and $\beta$ be any reals such that $\left(w_{\beta} / w_{\alpha}\right) \varphi_{c}$ is in $L_{\alpha}^{2}(0, \infty)$ and the following inequality in terms of the Section 2.2 growth measure $\delta_{\infty}$ holds:

$$
\frac{c}{a}<2 \delta_{\infty}(\psi)+\frac{1}{d} .
$$

Then we have the absolutely convergent Laguerre reduction series representation

$$
\mathrm{E}\left[\rho\left(Y_{d, h}\right)\right]=\chi\left(\frac{a}{c}\right) \sum_{n=0}^{\infty} a_{n}\left\langle\frac{w_{\beta}}{w_{\alpha}} \varphi_{c}, L_{n}^{\alpha}\right\rangle_{\alpha},
$$

whose remainder terms, $R_{N}=\chi(a / c) \sum_{n=N}^{\infty} a_{n}\left\langle\left(w_{\beta} / w_{\alpha}\right) \varphi_{c}, L_{n}^{\alpha}\right\rangle_{\alpha}$, satisfy

$$
R_{N}^{2} \leq\left\|\frac{w_{\beta}}{w_{\alpha}} \varphi_{c}\right\|_{\alpha}^{2} \chi^{2}\left(\frac{a}{c}\right) \sum_{n=N}^{\infty} a_{n}^{2}\left\|L_{n}^{\alpha}\right\|_{\alpha}^{2},
$$

recalling that $\left\|L_{n}^{\alpha}\right\|_{\alpha}^{2}=\Gamma(n+\alpha+1) / n$ !.

Here the $w_{\alpha}$ are the weight functions of Section 2.1, given by $w_{\alpha}(x)=x^{\alpha} \mathrm{e}^{-x}$, and the coefficients $a_{n}$ are given in terms of $\alpha, \beta, a$, and $c$ as linear combinations of $\psi$-twisted moments $\mathrm{E}\left[Y^{\kappa} \psi(Y)\right]$ of $Y \equiv Y_{d, h}$, as follows, setting $\delta_{k}=\alpha-\beta+k$ :

$$
a_{n}=\sum_{k=0}^{n} \frac{(-1)^{k}}{\Gamma(k+\alpha+1)}\left(\begin{array}{l}
n \\
k
\end{array}\right)\left(\frac{c}{a}\right)^{\delta_{k}} \mathrm{E}\left[Y^{\delta_{k}} \psi(Y)\right] .
$$

Briefly, the relevance of this result is that it reduces the computation of $\mathrm{E}[\rho(Y)]$ to the computation of the scalar products $\left\langle\left(w_{\beta} / w_{\alpha}\right) \varphi_{c}, L_{n}^{\alpha}\right\rangle_{\alpha}$ and the $\psi$-twisted moments $\mathrm{E}\left[Y^{\kappa} \psi(Y)\right]$. From Section 2.1, the scalar products are integrals of $\varphi_{c}$. Together with the $\psi$-twisted moments they may therefore be considered primitives of the problem.

\subsection{The second type of reduction series}

Working in the setting of Sections 5.1 and 5.2, we make explicit the second of the two types of Laguerre reduction series of Schröder (2006, Section 6) for the above stochastic functionals of $Y_{d, h}$. Recall that this series is in the spirit of Dufresne (2000) and constructs stochastic functionals as elements of Hilbert spaces using ladder height reduction series. For this we have to amend the setting of Section 5.1.

For any positive integer $N$, we make the following three additional assumptions. First, for some, and thus any, positive real $c$, let $\varphi_{c}$ be $(N-1)$-fold continuously differentiable on $(L(c), \infty)$. Second, assume that the $(N-1)$ th derivative, $\varphi_{c}{ }^{(N-1)}$, is a nonzero constant. Third, let $c$ be such that the inequalities

$$
0<\gamma_{A}\left(\varphi_{c}^{(\ell)}\right)+\ell+1+\gamma_{A}(\psi)(a / c), \quad A \in\{L(c), \infty\}
$$


hold for any $\ell$ in $\{0,1, \ldots, N-2\}$. With the proof postponed to Section 5.5, our precise result is then as follows.

Theorem 5.2. In the above setting, let the reals $\alpha>-1$ and $\beta$ be such that the following inequality in terms of the Section 2.2 growth measure $\delta_{\infty}$ holds:

$$
\frac{c}{a}<2 \delta_{\infty}(\psi)+\frac{1}{d}
$$

Then we have the ladder height reduction series representation

$$
\mathrm{E}\left[\rho\left(Y_{d, h}\right)\right]=w_{\beta}(L(c)) \sum_{n=0}^{\infty} b_{N, n} L_{n}^{\alpha}(L(c)),
$$

which is the evaluation at the point $L(c)$ of the element $\sum_{n=0}^{\infty} b_{N, n} L_{n}^{\alpha}$ of the Hilbert space $L_{\alpha}^{2}(0, \infty)$ and, furthermore, is absolutely convergent as a series of complex numbers.

Here $w_{\beta}$ is the weight function from Section 2.1 given by $w_{\beta}(x)=x^{\beta} \mathrm{e}^{-x}$, and the coefficients $b_{N, n}$ are given in terms of $\alpha, \beta, a, c$, and $N$ by linear combinations of $\psi$-twisted moments $\mathrm{E}\left[Y^{\kappa} \psi(Y)\right]$ of $Y=Y_{d, h}$, as follows:

$$
b_{N, n}=\sum_{k=0}^{n} \varphi_{c}^{(N-1)}\left(x_{0}\right) \frac{\alpha_{n, k}}{\left\|L_{n}^{\alpha}\right\|_{\alpha}^{2}} \chi\left(\frac{a}{c}\right) \frac{(c / a)^{N+\delta_{k}}}{\left(\delta_{k}+1\right)_{N}} \mathrm{E}\left[Y^{N+\delta_{k}} \psi(Y)\right] .
$$

In this expression, $x_{0}$ is any element of $(L(c), \infty)$, the quotients $\alpha_{n, k} /\left\|L_{n}^{\alpha}\right\|_{\alpha}^{2}$ are as in Section 2.1, the Pochhammer symbols $(\lambda)_{N}$ are as in Section 4, and we set $\delta_{k}=\alpha-\beta+k$.

\subsection{Determining the growth measures}

A principal first step towards establishing the reduction series of Sections 5.2 and 5.3 consists of determining the local growth measures of Section 2.2 for the densities, $f_{d, h}$, of $Y_{d, h}$. This is taken care of by the following result of Schröder (2005c), which may be regarded as a first, but typical, application of any of the available explicit representations of the law of the integral of geometric Brownian motion.

Theorem 5.3. Any density $f_{d, h}$ is an analytic function on the positive reals with growth orders $\gamma_{0}\left(f_{d, h}\right)=\infty, \gamma_{A}\left(f_{d, h}\right)=0$ for $A$ in $(0, \infty]$, and $\delta_{\infty}\left(f_{d, h}\right)=1 /(2 d)$.

Since this result is instrumental in implementing the general results of Schröder (2006) in the present situation, we sketch the key ideas of its proof from Schröder (2005c, Sections 5.3 and 5.4). We first reduce to the case $d=1$, where $f \equiv f_{1, h}$ is the density of $1 / A^{(v)}(h)$. From Schröder (2003) we have

$$
f(x)=\Gamma\left(-\mu_{v}\right) \frac{c(x)}{\pi} \mathrm{e}^{\pi^{2} /(2 h)} I\left(\sqrt{\frac{x}{2}}\right)
$$

for any positive real $x$, where $\mu_{v}=-(v+1)$ and

$$
\begin{aligned}
& c(x)=\frac{2^{v / 2}}{\sqrt{\pi h}} \mathrm{e}^{-v^{2} h / 2} x^{\mu_{v} / 2} \mathrm{e}^{-x / 2}, \\
& I(\xi)=\int_{0}^{\infty} \mathrm{e}^{-w^{2} /(2 h)} \sinh (w) \sin \left(\frac{\pi}{h} w\right) H_{\mu_{v}}(\xi \cosh (w)) \mathrm{d} w .
\end{aligned}
$$


Here $H_{\mu_{v}}$ is the Hermite function of degree $\mu_{v}$; see Lebedev (1972, Chapter 10). Using the analyticity of this density in $v$, now reduce to $v \neq-1$.

To prove that $\delta_{\infty}(f)=\frac{1}{2}$ it is then sufficient to show that $I(\sqrt{x / 2})$ can be bounded by a rational function in $x$ for $x \geq x_{0}$, where $x_{0} \gg 0$. To this end, the continuity of Hermite functions implies the existence of a constant, $C_{0}$, such that $\left|H_{\mu_{\nu}}(z)\right| \leq C_{0}$ for all complex $z$ in the closed disc of radius $w_{0} \gg 0$ centred at 0 . On the other hand, enlarging $C_{0}$ if necessary, $\left|H_{\mu_{v}}(z)\right| \leq C_{0}|z|^{\mu_{v}}$ for $|z| \geq w_{0}$, using the asymptotic expansion for Hermite functions of Lebedev (1972, Section 10.6). Breaking the integral $I(\sqrt{x / 2})$ at $w_{0}$ thus gives

$$
\begin{aligned}
0 \leq I\left(\sqrt{\frac{x}{2}}\right) \leq & C_{0} \int_{0}^{w_{0}} \mathrm{e}^{-w^{2} /(2 h)} \sinh (w) \mathrm{d} w \\
& +C_{0}\left(\frac{x}{2}\right)^{\mu_{\nu} / 2} \int_{w_{0}}^{\infty} \mathrm{e}^{-w^{2} /(2 h)} \sinh (w) \cosh ^{\mu_{\nu}}(w) \mathrm{d} w
\end{aligned}
$$

and, hence, the desired majorizing rational function of $x \gg 0$.

Proving that $\gamma_{0}(f)=\infty$ reduces to showing that, for any nonnegative integer $k$, the $k$ th derivative of $I$ is 0 at $\xi=0$. Taking derivatives of $I$ means taking them under the integral sign. Using the derivative rule $(\mathrm{d} / \mathrm{d} z) H_{-\mu}(z)=-2 \mu H_{-(\mu+1)}(z)$ for Hermite functions of Lebedev (1972, Equation (10.4.4)), the problem therefore reduces on inspection to showing that

$$
0=\int_{\mathbb{R}} \mathrm{e}^{-y^{2} / 2} \cosh ^{k}(\sqrt{h} y)\left(\mathrm{e}^{\sqrt{h} y+\mathrm{i} y \pi / \sqrt{h}}-\mathrm{e}^{\sqrt{h} y-\mathrm{i} y \pi / \sqrt{h}}\right) \mathrm{d} y
$$

for any nonnegative integer $k$. This can now be seen by an argument essentially along the lines of that in Yor (1992a, p. 529).

Since $f$ is continuous and nonzero on the positive reals, we finally have $\gamma_{A}(f)=0$ at any $A$ in $(0, \infty)$, and the proof of Theorem 5.3 is complete.

\subsection{Proofs of Theorems 5.1 and 5.2}

We establish the two reduction series of Theorems 5.1 and 5.2 together. Instrumental in this is the Theorem 5.3 determination of the growth measures of the density, $f_{d, h}$, of any $Y_{d, h}$. With this information, Theorem 5.1 is obtained by specializing Schröder (2006, Theorem 6.1) to $Y=Y_{d, h}$ and Theorem 5.2 is obtained by specializing Schröder (2006, Theorem 6.2) to this situation.

To exemplify the arguments, we establish the second of these results. Letting $f \equiv f_{d, h}$, first consider the additional inequalities to be satisfied for Schröder (2006, Theorem 6.2) to hold, namely

$$
0<\gamma_{A}\left(\varphi_{c}^{(\ell)}\right)+\ell+1+\left(\gamma_{A}(\psi)+\gamma_{A}(f)\right)(a / c), \quad A \in\{L(c), \infty\},
$$

for all $\ell$ in $\{0,1, \ldots, N-2\}$. They simplify to those required for Theorem 5.2 to hold, recalling from Theorem 5.3 that $\gamma_{A}(f)=0$ for $A$ in $(0, \infty)$. The second of the two inequalities to be satisfied in Schröder (2006, Theorem 6.2), namely

$$
\max \{2 \beta-(2 N+\alpha+1),-2\}<2\left(\gamma_{0}(\psi)+\gamma_{0}(f)\right)(a / c),
$$

holds without restriction because of the Theorem 5.3 result $\gamma_{0}(f)=\infty$. Upon substitution of the Theorem 5.3 result $\delta_{\infty}(f)=1 /(2 d)$, the first of the inequalities of Schröder (2006, Theorem 6.2), on the other hand, is just the single inequality of Theorem 5.2, and the proof is complete. 


\section{Laguerre reduction series for the Asian option value functionals}

In this section we illustrate the ladder height Laguerre reduction series of Section 5 in the option valuation context. The focus is on Asian option valuation in Brownian motion models, an issue which has motivated much of the development in this area. The discussion proceeds in two steps, leading to the reduction series of Section 6.2 as its main results.

\subsection{Asian options}

There is by now a standard framework for the analysis of Asian options; see Carr and Schröder (2004), following Geman and Yor (1993). However, it needs to be modified in order to apply to this paper's Laguerre reduction approaches. We address these modifications in this section, with Lemmas 6.2 and 6.3 below as the key results. Using risk-neutral valuation, we work in the Black-Scholes framework with two securities. First, there is a riskless security, a bond, whose price grows at the continuously compounding positive interest rate $r$. Second, there is a risky security, whose price process, $S$, is modelled as follows. Consider a complete probability space equipped with the standard filtration of a standard Brownian motion on the time set $[0, \infty)$. On this filtered space, we have the risk-neutral measure $Q$, the probability measure in use in the sequel, and a standard $Q$-Brownian motion $W$ such that

$$
S_{t}=S_{0} \mathrm{e}^{2 \xi_{t}}, \quad \text { where } 2 \xi_{t}=\mu t+\sigma W_{t},
$$

for any real $t \geq 0$. Here $S_{0}$ and the volatility $\sigma$ are positive reals and $\mu=\varpi-\frac{1}{2} \sigma^{2}$ for any real $\varpi$. The specific form of the otherwise arbitrary risk-neutral drift $\varpi$ depends on the nature of the security modelled. For example, if $S$ is a stock it is the interest rate $r$ minus the pertinent dividend rate.

The European-style arithmetic-average Asian option written at time $t_{0}$, with maturity $T$ and fixed strike price $K$, is then the contingent claim on the closed time interval $\left[t_{0}, T\right]$ with the following pay-off at time $T$ : the maximum of 0 and the excess over $K$ of the average price, $\left(1 /\left(T-t_{0}\right)\right) \int_{\left[t_{0}, T\right]} S_{u} \mathrm{~d} u$, over the period $\left[t_{0}, T\right]$. The price, $C_{t}$, of the Asian option at any time $t$ between $t_{0}$ and $T$ is thus given by the risk-neutral expectation

$$
C_{t}=\mathrm{e}^{-r(T-t)} \mathrm{E}\left[\left(\frac{1}{T-t_{0}} \int_{t_{0}}^{T} S_{u} \mathrm{~d} u-K\right)^{+} \mid \mathcal{F}_{t}\right],
$$

which is conditional on the information $\mathcal{F}_{t}$ available at time $t$. Elaborating on the modification in Carr and Schröder (2004, Part I) of a technique of Geman and Yor (1993), the idea is to construct stochastic functionals $C^{(v)}(h)$ which afford a factorization

$$
C_{t}=\frac{\mathrm{e}^{-r(T-t)}}{T-t_{0}} \frac{4 S_{t}}{\sigma^{2}} C^{(v)}(h)
$$

and each take the role of a normalized price. They depend on the dimensionless parameters

$$
v=\frac{2 \varpi}{\sigma^{2}}-1 \quad \text { and } \quad h=\frac{\sigma^{2}}{4}(T-t),
$$

where $v$ is the normalized adjusted interest rate and $h$ is the normalized time to maturity, which is nonnegative. The functionals are in terms of the integral of geometric Brownian motion $A_{h}^{(v)}$; recall from Section 5.1 that these processes are given by $A_{x}^{(v)}=\int_{0}^{x} \exp \left(2\left(v u+W_{u}\right)\right) \mathrm{d} u$ for 
any real $x>0$, with $W$ denoting Brownian motion. The exact form of the functionals is then determined by a parameter $q$ which is given, as a function of $h$ in particular, by

$$
q=\frac{T-t_{0}}{(2 / \sigma)^{2} S_{t}}\left(K-\frac{1}{T-t_{0}} \int_{t_{0}}^{t} S_{u} \mathrm{~d} u\right),
$$

and can be thought of as a normalized strike price. If $q$ is nonpositive, Asian options lose their option feature and, as has already been observed in Geman and Yor (1993, p. 361), we have the following lemma, which is almost tautological.

Lemma 6.1. If $q \leq 0$ then $C^{(v)}(h)=\mathrm{E}\left[A_{h}^{(v)}\right]-q$, where $\mathrm{E}\left[A_{h}^{(v)}\right]=\left(\mathrm{e}^{2 h(v+1)}-1\right) /(2(v+1))$, as an analytic function of $v$.

Valuation of Asian options thus reduces to the case $q>0$. It is in this situation that the results of Sections 5.2 and 5.3 are to be applied, to compute $C_{t}$. However, since the latter is an expectation in $A^{(v)}$, it must be re-expressed in terms of the reciprocal of such processes for the Laguerre reduction approaches to be applicable. A principal renormalization technique to address this problem was developed in Schröder (2005c, Section 4), and here Schröder (2005c, Lemma 7.2) gives the following result.

Lemma 6.2. If $q>0$ then $C^{(v)}(h)=\mathrm{E}\left[A_{h}^{(v)}\right]-q+\mathrm{E}\left[\left(\psi h_{c}\right)\left(Y_{c q, h}\right)\right]$, with the functions $\psi(x)=q x^{-1}$ and $h_{c}(x)=(x-c)^{+}$defined on the positive real line.

Here, recall from Section 5 that $Y_{d}$, for any positive real $d$, is the reciprocal of the integral of geometric Brownian motion, the process given by $Y_{d, h}=d / A^{(v)}(h)$ for any real $h>0$.

Valuing any Asian option is thus reduced to computing a single function, the normalized price functional $C^{(v)}$. Referring to Schröder (2005c, Section 7.2), this enables a one-pricefor-all valuation of Asian options as a practical consequence. Since we have $C^{(v)}(h)=$ $\mathrm{E}\left[\left(A^{(v)}(h)-q\right)^{+}\right]$, irrespective of the sign of $q$, this reduction is also in accordance with that of Carr and Schröder (2004, Part I), following Geman and Yor (1993). The point is that this representation now enables us to apply the results of Section 5. The basis of this is the following observation.

Lemma 6.3. For any positive reals $d$ and $c$, the triple $\left(\psi, h_{c}, Y_{d, h}\right)$ is admissible in the sense of Section 5.1 with $\chi$ and $L$ there equal to the identity mapping on the positive reals.

In fact, the first moment of $A^{(v)}(h)$ is finite and positive as a consequence of the explicit formula for it in Lemma 6.1. The properties in terms of the functions $\chi$ and $L$ to be checked are clear on inspection, so the proof of Lemma 6.3 is complete.

\subsection{Ladder height reduction series for valuing Asian options}

Working in the setting of Section 6.1, analysing the normalized call price functionals $C^{(v)}$ of Asian options reduces to analysing the put price functionals $P^{(v)}$ via the identities

$$
C^{(v)}(h)=\mathrm{E}\left[A_{h}^{(v)}\right]-q+P^{(v)}(h),
$$

where, for any real $c>0$,

$$
P^{(v)}(h)=\mathrm{E}\left[\left(\psi h_{c}\right)\left(Y_{c q, h}\right)\right]
$$

with $\psi(x)=q / x$ and $h_{c}(x)=(x-c)^{+}$. Postponing proofs to the end of this section, we now discuss our reduction series for these functionals. We start with those of the second type, developed in Section 5.3, which replicate Dufresne (2000, Theorem 7.1) under less restrictive conditions. 
Theorem 6.1. For any reals $\alpha>-1$ and $\beta$ and any positive real $c$ such that $0<c q<1$, we have the absolutely convergent ladder height reduction series representation

$$
P^{(v)}(h)=c^{\beta} \mathrm{e}^{-c} \sum_{n=0}^{\infty} c_{2, n} L_{n}^{\alpha}(c),
$$

whose $\alpha$-Laguerre coefficients $c_{2, n}$, on setting $\delta_{k}=\alpha-\beta+k$, are given by

$$
c_{2, n}=q \sum_{k=0}^{n} \frac{(-1)^{k}}{\Gamma(k+\alpha+1)}\left(\begin{array}{l}
n \\
k
\end{array}\right)\left(\frac{c q}{h}\right)^{\delta_{k}+1} \frac{\mathrm{E}\left[Y_{h, h}^{\delta_{k}+1}\right]}{\left(\delta_{k}+1\right)\left(\delta_{k}+2\right)} .
$$

Notice how this reduction series is obtained by 'linear combination' of the moments of $Y_{h, h}$, with coefficients in terms of elementary functions and the gamma function only.

Providing workable estimates for the approximation errors of this series, as would be highly desirable, seems to be highly problematic, however. For the first type of reduction series, in contrast, such an explicit error analysis can be established as a new feature, as follows.

Theorem 6.2. For any reals $\alpha>-1$ and $\beta$ and any positive real $c$ such that $0<c q<1$, we have the absolutely convergent ladder height Laguerre reduction series representation

$$
P^{(v)}(h)=\sum_{n=0}^{N} c_{0, n} \sum_{k=0}^{n}\left(\Gamma\left(\beta_{k+2}, c\right)-c \Gamma\left(\beta_{k+1}, c\right)\right) \alpha_{n, k}+R_{N},
$$

where $\beta_{m}=\beta+m$, whose $\alpha$-Laguerre coefficients are given by

$$
c_{0, n}=q \sum_{k=0}^{n} \frac{(-1)^{k}}{\Gamma(k+\alpha+1)}\left(\begin{array}{l}
n \\
k
\end{array}\right)\left(\frac{c q}{h}\right)^{\delta_{k}-1} \mathrm{E}\left[Y_{h, h}^{\delta_{k}-1}\right],
$$

where $\delta_{k}=\alpha-\beta+k$. Its error terms, $R_{N}$, satisfy

$$
R_{N}^{2} \leq D_{\alpha}(\beta) \sum_{n=N+1}^{\infty} c_{0, n}^{2}\left\|L_{n}^{\alpha}\right\|_{\alpha}^{2}
$$

where $D_{\alpha}(\beta)=\Gamma(b+1, c)-2 c \Gamma(b, c)+c^{2} \Gamma(b-1, c)$ with $b=2(\beta+1)-\alpha$, recalling that $\left\|L_{n}^{\alpha}\right\|_{\alpha}^{2}=\Gamma(n+\alpha+1) / n$ !.

Here the $\alpha_{n, k}=(-1)^{k}\left(\begin{array}{c}n+\alpha \\ n-k\end{array}\right) / k$ ! (from Section 2.1) are the coefficients of the $\alpha$-Laguerre polynomials $L_{n}^{\alpha}$ and the reduction series is in terms of the complementary incomplete gamma function, $\Gamma(s, x)$. The above Laguerre reduction series is thus also obtained by 'linear combination' of the moments of $Y_{h, h}$.

Proofs of Theorems 6.1 and 6.2. We prove the theorems by reduction to the results of Section 5. These are to be applied with the parameters $d=c q$ and $a=c$, with the functions $\varphi_{c}=h_{c}$ and $\psi(x)=q / x$, and with both $\chi$ and $L$ the identity mapping on the positive reals.

The proof of Theorem 6.1 is then obtained by specializing Theorem 5.2 to $N=2$, and essentially reduces to checking three inequalities. Indeed, the $\gamma_{A}$-growth measure inequalities for Theorem 5.2 now amount to $0<\gamma_{A}\left(h_{c}\right)+1+\gamma_{A}(\psi)$ for $A$ in $\{c, \infty\}$. The inequality for $A=c$ is true since $h_{c}(c)=0$, and that for $A=\infty$ holds because the $\gamma_{\infty}$ s cancel. Next, using 
$\delta_{\infty}(\psi)=0$, the inequality $1<2 \delta_{\infty}(\psi)+1 /(c q)$ of Theorem 5.2 is seen to specialize to that of Theorem 6.1. With $\varphi_{c}$ having support away from 0 , the map $\left(w_{\beta} / w_{\alpha}\right) \varphi_{c}$ is in $L_{\alpha}^{2}(0, \infty)$ for any choice of $\alpha>-1$ and $\beta$. Now specialize the coefficients in Theorem 5.2, recalling that $\chi$ is the identity mapping and that $N=2$. The proof of Theorem 6.1 is thus complete.

The proof of Theorem 6.2 is obtained by further specializing Theorem 5.1 to $N=0$. Then any coefficient integral $\left\langle\left(w_{\beta} / w_{\alpha}\right) \varphi_{c}, L_{n}^{\alpha}\right\rangle_{\alpha}$ can be checked to be equal to

$$
\sum_{k=0}^{n} \alpha_{n, k}\left(\Gamma\left(\beta_{k+2}, c\right)-c \Gamma\left(\beta_{k+1}, c\right)\right)
$$

there, which completes the proof of Theorem 6.2.

\section{Moments of the integral of geometric Brownian motion}

In the previous two sections we have exemplified how the general theory of Schröder (2006) is principally able to reduce the study of the Section 5.1 stochastic functionals to that of the moments of the pertinent processes: the integral of geometric Brownian motion $A^{(v)}$, for any real $\nu$, and its reciprocals $Y_{a}$, for any positive real $a$. From Section 5.1, for any real $h$ these processes are given by

$$
Y_{a, h}=\frac{a}{A_{h}^{(v)}}, \quad \text { where } A_{h}^{(v)}=\int_{0}^{h} \mathrm{e}^{2\left(v u+W_{u}\right)} \mathrm{d} u,
$$

and for this first step of the reduction we require additional information specific to the processes, namely that of the explicit asymptotics of the law of $A^{(v)}$.

As the final step of our two-step approach, in this section we develop the structure of the integral moments of $A^{(v)}$. Our results were referred to in Schröder (2005c), where they were shown to provide most efficient methods for numerical computation. Our approach can thus be considered constructive, and it seems apt to precede the structure theory with a summary of the results slanted towards this theme.

\subsection{Statement and discussion of main results with a view to computability}

In this section we state and put into perspective one of this paper's main results, our explicit formulae for the negative moments of $A^{(v)}$. Indeed, the moments of $A^{(v)}$ have been thoroughly studied; see, for instance, Dufresne (1989), Yor (1997), and the references therein. They are stated explicity in the following theorem.

Theorem 7.1. For any positive real $h$ and any nonnegative integer $n$, we have

$$
\mathrm{E}\left[\left(A_{h}^{(v)}\right)^{n}\right]=\frac{n !}{2^{2 n}} \sum_{k=0}^{n} c_{k}\left(\frac{v}{2}\right) \mathrm{e}^{2 h k(k+v)},
$$

where

$$
c_{k}(\mu)=\prod_{\ell \neq k} \frac{2}{(\mu+\ell)^{2}-(\mu+k)^{2}},
$$

the product running over all nonnegative integers $\ell$ less than or equal to $n$. 
This is Yor (1992a, Equation (4.d')) with a direct proof in Yor (1992a, pp. 519f.). Further comments on its history can be found in Yor (2001, Postscript \#3b), p. 54).

The negative moments of $A^{(v)}$ are of a different nature. Determining their structure and giving explicitly computable formulae for them are main contributions of the paper. Our characterization is in two steps, which are explained in Theorems 7.2 and 7.3 below. The first step reduces the computation of negative moments to that of functions $\Theta_{k}$, as follows.

Theorem 7.2. For any positive integer $n$, we have

$$
\mathrm{E}\left[Y_{h, h}^{n}\right]=\exp \left(-\frac{v^{2} h}{2}\right) \sum_{k=1}^{n} a_{n, k}^{*}\left(2 h^{k} \Theta_{k}(h)+\sum_{\ell=0}^{\ell_{v}-1} \Delta_{k}^{*}\left(a_{\ell}, h\right)\right),
$$

where $\ell_{v}$ is the smallest nonnegative integer $\ell$ such that $a_{\ell}=2 \ell+1-|v-1|$ is nonnegative and $\Delta_{k}^{*}$ is given by

$$
\Delta_{k}^{*}(a, h)=\frac{1}{k-\frac{1}{2}} A_{-1 / 2}(h)-\frac{h|a|}{k-\frac{1}{2}} G_{k-1}^{*}\left(b_{a} h\right)-2 h a \frac{\left(-b_{a} h\right)^{k-1}}{\left(\frac{1}{2}\right)_{k}} \mathrm{e}^{b_{a} h}-\frac{2}{|a|} G_{k}^{*}\left(b_{a} h\right)
$$

for any negative real $a$ and any positive real $h$, on setting $b_{a}=a^{2} / 2$.

To explain the notation here, first recall the Pochhammer symbol $(\lambda)_{k}$ of any complex $\lambda$, defined by $(\lambda)_{0}=1$ and $(\lambda)_{k+1}=(\lambda-k)(\lambda)_{k}$ for any integer $k \geq 0$. Then we have

$$
a_{n, k}^{*}=(-1)^{k-1}\left(\frac{1}{2}\right)_{k} h^{n-k} a_{n, k},
$$

where $a_{n, k}$ are reals recursively defined by $a_{1,1}=1$ and, for any integer $n \geq 1$, by

$$
a_{n+1,1}=\beta_{n} a_{n, 1}, \quad a_{n+1, n+1}=\alpha_{n} a_{n, n}, \quad a_{n+1, k}=\beta_{n} a_{n, k}+\alpha_{n} a_{n, k-1}
$$

for any integer $k$ in $\{2, \ldots, n\}$, setting $\alpha_{n}=-1 / n$ and $\beta_{n}=2(n-v)+v^{2} /(2 n)$. They have the combinatorial description

$$
a_{n+1, k+1}=\sum_{J_{k}} \alpha_{J_{k}} \beta_{J_{k}^{\mathrm{c}}}
$$

for any nonnegative integers $n$ and $k \leq n$. Here the sum is over all $k$-tuples $J_{k}=\left(j_{1}, \ldots, j_{k}\right)$ of strictly increasingly ordered integers $j_{\ell}$ in $\{1, \ldots, n\}$, and $J_{k}^{\mathrm{c}}=\left(\ell_{1}, \ldots \ell_{n-k}\right)$ is the complement of $J_{k}$ defined by $\left\{\ell_{1}, \ldots, \ell_{n-k}, j_{1}, \ldots, j_{k}\right\}=\{1, \ldots, n\}$. We will use subindexing by a $k$-tuple to indicate products as follows: $\alpha_{J_{k}}=\alpha_{j_{1}} \alpha_{j_{2}} \cdots \alpha_{j_{k}}$. The empty product is equal to 1 .

The functions $A_{a}$, for any real $a$, and $G_{k}^{*}$, for any integer $k \geq 1$, are those defined in Section 4; in particular, recall how the latter functions are given, as weighted complementary incomplete gamma functions, by $G_{k}^{*}(x)=(1 / \sqrt{\pi}) x^{k} \mathrm{e}^{x} \Gamma\left(\frac{1}{2}-k, x\right)$ for any real $x>0$.

With an explicit formula for $\Theta_{k}$ in Theorem 7.3, we postpone a discussion of these functions in favour of a broad outline of the ideas upon which Theorem 7.2 is based. In fact, the line of reasoning leading to this last result proceeds in two steps as well. As recalled in Section 7.2, functional recurrence rules have been derived for negative moments in Dufresne (2000). Studying a somewhat more general problem, we solve them in Section 7.3 as a first step, and thus express any $-n$th moment of $A^{(v)}$ as a linear combination of the first $n$ derivatives of the -1 th moment of $A^{(v)}$ considered as a function in time. These are studied in Sections 7.4 to 7.7 as a second step, and, in combination with the results of the first step, enable us to 
prove Theorem 7.2 in Section 7.8. From a more structural point of view, we thus in fact find two integral representations for the negative moments of $A^{(v)}$; an obvious one, described in Theorem 7.6, and an unexpected one, described in Theorem 7.2. To develop the latter we proceed by interpreting negative moments in terms of theta functions, reviewed in Section 3.1. This may qualify as a novel point of view, and the general idea of this 'modular interpretation' is that negative moments of $A^{(v)}$ are linear combinations of particular integrals of theta functions, namely the functions $\Theta_{k}$ for any integer $k \geq 1$. More precisely, any of these theta integrals is, for any positive real $h$, given by

$$
\Theta_{k}(h)=\int_{0}^{\infty} \vartheta\left(\frac{v}{2} \mid w\right) Q_{k}(h, w) \mathrm{d} w,
$$

where, from Section 4.1,

$$
Q_{k}(h, w)=\frac{(2 w)^{k-1}}{(2 h w+1)^{k+1 / 2}} .
$$

However, depending on $v$, only a part of the negative moments can in general be identified in this way, and correcting functions are needed. The particular case where no such correcting functions are needed is the subject of the first of the two remarks to follow.

Remark 7.1. For $|v-1| \leq 1$, Theorem 7.2 asserts that any negative moment of $A^{(v)}$ is obtained from theta integrals only:

$$
\mathrm{E}\left[\left(A_{h}^{(v)}\right)^{-n}\right]=\exp \left(-\frac{v^{2} h}{2}\right) \sum_{k=1}^{n}(-1)^{k-1}\left(\frac{3}{2}\right)_{k-1} a_{n, k} \Theta_{k}(h) .
$$

Note that in the zero-drift case, $v=0$, a different representation for the above expectations has been obtained in Donati-Martin et al. (2000a, Section 5):

$$
\mathrm{E}\left[\left(A_{h}^{(0)}\right)^{-n}\right]=\frac{2^{n+1}}{\Gamma(n)} \int_{0}^{\infty} \mathrm{e}^{-w^{2} h / 2} P_{n}(w) \frac{\mathrm{d} w}{w},
$$

where

$$
P_{n}(w)=\prod_{k=0}^{n-1}\left(k^{2}+(w / 2)^{2}\right)
$$

The integrals of Theorem 7.6 can be checked to replicate this representation, but it is a theorem that, for $v=0$, the formula in Theorem 7.2 specializes to it as well. In this sense, the alternative approach developed in this paper enables us, in particular, to handle the effects of nonzero drift terms: increasing $v$ in absolute value will introduce the correction terms $\Delta_{k}^{*}$ in Theorem 7.2.

Remark 7.2. The occurrence of theta functions is well known in connection with studying Brownian motion on a finite interval; see, for instance, Karatzas and Shreve (1991, Section 2.8C). As a modification of Exercise 8.9 there, consider, for any positive real $a$, the function $\varphi_{a}$, on the reals, that satisfies $\varphi_{a}(n a)=a$ for any integer $n$ and linearly connects 0 with this value on the open interval $(n a,(n+1) a)$, in the sense that $\varphi_{a}(x)=x-n a$ there. The law of the doubly reflected Brownian motion $\varphi_{a}(W)$ at any time $t$ can then be checked to be given in terms of theta functions as follows, for any real $x$ :

$$
\operatorname{law}\left(\varphi_{a}(W)\right)(t, x)=\frac{1}{a} \mathbf{1}_{[0, a]}(x) \vartheta\left(\frac{x}{a} \mid \frac{2 t}{a^{2}}\right) .
$$


From the heat equation perspective of Section 3.1, these are representations in terms of the state space variable $x$, whereas in the present context it is time which becomes a variable in the theta integrals. Moreover, as Remark 7.1 clearly shows, Theorem 7.2 affords a representation of a quantity involving integration over a finite time interval by finitely many integrals over the whole (infinite) time interval from time 0 to time $\infty$.

Taking up the computational aspects of Theorem 7.2, the $\Delta_{k}^{*}$ are given in terms of standard functions. Thus, the question that remains is that of how to compute the theta integrals $\Theta_{k}$. This is the second main step of our analysis of the negative moments of $A^{(v)}$, and we here discuss three of our series in this context. The first of these is in terms of elementary functions and the error integral. It is also uniformly applicable and can be optimized with respect to convergence speed as follows.

Theorem 7.3. For any $B$ in the extended nonnegative reals, $[0, \infty]$, we have the representation $\Theta_{n}=F_{B}+I_{B}$, where the functions $F_{B}$ and $I_{B}$ are given by the absolutely convergent series

$$
\begin{aligned}
F_{B}(h) & =\sum_{m \in \mathbb{Z}} C_{|2 m+v|, n}(B, h), \\
I_{B}(h) & =\sum_{m=0}^{\infty} \varepsilon_{m} \cos (\pi m v) D_{\pi m, n}(B, h),
\end{aligned}
$$

for any positive real $h$, where $\varepsilon_{0}=1$ and $\varepsilon_{m}=2$ for any positive integer $m$.

Here the functions $C_{a, n}$ and $D_{a, n}$ are those defined in Section 4.1 and shown in Section 4.2 to be finite linear combinations of weighted complementary incomplete gamma functions.

The structural properties of theta functions are essential for this result, whose proof is given in Section 7.10. From a computational point of view, introducing the parameter $B$ enables us to optimize the convergence speed of these series. In fact, by defining error terms $R_{k, M}(B, h)$ via

$$
\begin{aligned}
F_{B}(h) & =\sum_{|m| \leq M} C_{|2 m+v|, n}(B, h)+R_{1, M}(B, h), \\
I_{B}(h) & =\sum_{m=0}^{M} \varepsilon_{m} \cos (\pi m v) D_{\pi m, n}(B, h)+R_{2, M}(B, h),
\end{aligned}
$$

for any integer $M \geq 1$, from Schröder (2005c, Section 6.10) we have the following result.

Proposition 7.1. For any positive real $B$,

$$
0<R_{1, M}(B, h) \leq \frac{2^{-1 / 2}}{h^{n+1 / 2}}\left(\frac{\mathrm{e}^{-v_{M}^{2} / B}}{v_{M} \sqrt{\pi}}-\frac{1}{\sqrt{B}} \operatorname{Erfc}\left(\frac{v_{M}}{\sqrt{B}}\right)\right),
$$

if $M$ is such that $v_{M}=M-|v / 2| \neq 0$, and

$$
\left|R_{2, M}(B, h)\right| \leq \frac{1}{h^{n-1}} \frac{2}{\sqrt{\pi}}\left(\frac{\mathrm{e}^{-(\pi M)^{2} B}}{\pi M \sqrt{\pi}}-\sqrt{B} \operatorname{Erfc}(\pi M \sqrt{B})\right) .
$$

These majorizations show that as $M$ tends to $\infty$ the error terms $R_{1, M}(B, h)$ tend to 0 with at least the squared exponential rate $(M-|\nu / 2|)^{2} / B$, and that the error terms $R_{2, M}(B, h)$ tend 
to 0 with at least the squared exponential rate $(\pi M)^{2} B$. In the extreme cases $F_{\infty}$ and $I_{0}$, in contrast, we have polynomial-order convergence to 0 only. As we will show in Section 7.10, the two series thus obtained are expressible in terms of Kummer's confluent hypergeometric function $\Psi$ (see Lebedev (1972, Chapter 9)), as follows.

Theorem 7.4. We have the absolutely convergent series

$$
h^{n} \Theta_{n}(h)=\frac{1}{\sqrt{2 \pi h^{-1}}} \sum_{m \in \mathbb{Z}} \Psi\left(1, \frac{3}{2}-n ; 2 h\left(m+\frac{v}{2}\right)^{2}\right),
$$

with good convergence behaviour for small values of $n$, and

$$
h^{n} \Theta_{n}(h)=\Gamma(n) \frac{1}{2} \Psi\left(n, \frac{1}{2} ; 0\right)+\Gamma(n) \sum_{m=1}^{\infty} \cos (\pi m \nu) \Psi\left(n, \frac{1}{2} ; \frac{(\pi m)^{2}}{2 h}\right),
$$

with good convergence behaviour for large values of $n$.

While these representations are very pleasing from an aesthetical point of view, the complexity of the confluent hypergeometric functions may however render the last two series not best suited for actual computation. Moreover, we must be very careful in choosing which of the two series is appropriate for the problem. Series similar to the first one of Theorem 7.4 have been derived in Donati-Martin et al. (2002, Section 4) in the zero-drift case, $v=0$, in a different context and for apparently different purposes.

\subsection{Preliminaries and recollections about negative moments}

In this section we collect preliminaries about the negative moments $m_{n}^{+}$given, for any positive integer $n$, by

$$
m_{n}^{+}(h)=\exp \left(\frac{v^{2} h}{2}\right) \mathrm{E}\left[\left(A_{h}^{(v)}\right)^{-n}\right], \quad h \in(0, \infty) .
$$

They have their origins in the idea of studying the structure of $A^{(v)}$ in terms of beta and gamma variables. The generalized moments, the expectations $\mathrm{E}^{Q}\left[\left(A^{(v)}(h)\right)^{r}\right]$ with $r$ any nonnegative real, have received much attention. Yor found their Laplace transforms with respect to time $h$ to be quotients of gamma values; see Yor (1992b, Equation (6.3), p. 69). In Dufresne (2000, Section 4), this result of Yor was extended to negative moments. The precise result of Dufresne, the proof of which is given below, is as follows.

Lemma 7.1. For any real $v$, we have the integral representation

$$
m_{1}^{+}(h)=\frac{2}{\sqrt{2 \pi h^{3}}} \int_{0}^{\infty} y \mathrm{e}^{-y^{2} /(2 h)} \frac{\cosh ((v-1) y)}{\sinh (y)} \mathrm{d} y, \quad h \in(0, \infty) .
$$

The higher negative moments of $A^{(v)}$ are determined recursively from this. Considering them as functions in time $h>0$, the precise result of Dufresne is as follows.

Lemma 7.2. For any positive integer $n$, we have $m_{n+1}^{+}=\beta_{n} m_{n}^{+}+\alpha_{n}\left(m_{n}^{+}\right)^{\prime}$, setting $\alpha_{n}=-1 / n$ and $\beta_{n}=2(n-v)+v^{2} /(2 n)$.

This is proved using the Itô lemma and applying time reversal. Different angles on these results of Dufresne have been developed in Donati-Martin et al. (2000a), (2000b), (2002). 
Proof of Lemma 7.1. We give a proof of Dufresne's formula for $m_{1}^{+}$of Lemma 7.1 which is based on ideas of Donati-Martin et al. (2002) and uses the explicit form of the law of $A^{(v)}$ derived in Yor (1992a, Section 6). Indeed, we apply Girsanov's theorem such that $B_{h}=v h+W_{h}$ becomes a standard Brownian motion, reduce to the drift-0 process $A=A^{(0)}$, and then condition on the Brownian motion to obtain

$$
m_{1}^{+}(h)=\mathrm{E}\left[\mathrm{e}^{v B_{h}} \frac{1}{A_{h}}\right]=\int_{-\infty}^{\infty} \frac{\mathrm{e}^{-x^{2} /(2 h)}}{\sqrt{2 \pi h}} \mathrm{e}^{v x} \mathrm{E}\left[\frac{1}{A_{h}} \mid B_{h}=x\right] \mathrm{d} x .
$$

By breaking the integral at 0 and expressing the integration over the negative real line from $-\infty$ to 0 as an integral over the positive real line from 0 to $\infty$, the proof of Dufresne's formula for $m_{1}^{+}$is seen to reduce to showing that

$$
\mathrm{E}\left[\frac{1}{A_{h}} \mid B_{h}=x\right]=\frac{x}{h} \frac{\mathrm{e}^{-x}}{\sinh (x)}=\frac{x}{h}(\operatorname{coth}(x)-1)
$$

for any positive real $x$. Following the ideas leading to Donati-Martin et al. (2002, Proposition 4.2), for any positive real $x$ we compute, as a first step, the expectations

$$
\mathrm{E}(\xi)=\frac{\mathrm{e}^{-x^{2} /(2 h)}}{\sqrt{2 \pi h}} \mathrm{E}\left[\exp \left(-\frac{\xi}{A_{h}}\right) \mid B_{h}=x\right],
$$

for any nonnegative real $\xi$. This computation is based on the integral representation of the conditional law $\mathrm{P}\left(A_{h} \in \mathrm{d} u \mid B_{h}=x\right)$ derived in Yor (1992a). We use this representation in the form of Schröder (2003, Lemma 4.1, p. 169), where it was expressed as a multiple of the Laplace inverse of an $I$-Bessel function as follows:

$$
\mathrm{P}\left(A_{h} \in \mathrm{d} u \mid B_{h}=x\right)=\frac{1}{u} \exp \left(-\frac{1+\mathrm{e}^{2 x}}{2 u}\right) \mathcal{L}^{-1}\left(I_{\sqrt{2 z}}\left(\frac{\mathrm{e}^{x}}{u}\right)\right)(h) \mathrm{d} u .
$$

Changing variable according to $w u=\mathrm{e}^{x}$ thus gives, on applying Fubini's theorem,

$$
\mathrm{E}(\xi)=\mathcal{L}^{-1}\left(\int_{0}^{\infty} \mathrm{e}^{-a w} I_{\sqrt{2 z}}(w) \frac{\mathrm{d} w}{w}\right)(h)
$$

for $\operatorname{Re}(z) \gg 0$, where

$$
a \equiv a(x, \xi)=\frac{\xi}{\mathrm{e}^{x}}+\cosh (x) .
$$

The integral to be Laplace inverted is a particular case of the Lipschitz-Hankel integrals discussed in Watson (1944, 13.2), and from Watson (1944, Equation (1), p. 387) it is expressible in terms of Legendre functions. A direct argument can be given using elementary properties of the Gauss hypergeometric function ${ }_{2} F_{1}$. In fact, expanding the Bessel function factor of the integrand, a term-by-term integration yields

$$
\int_{0}^{\infty} \mathrm{e}^{-a w} I_{\mu}(w) \frac{\mathrm{d} w}{w}=\frac{1}{\mu} \frac{1}{(2 a)^{\mu}}{ }_{2} F_{1}\left(\frac{\mu}{2}, \frac{\mu+1}{2} ; \mu+1 ; \frac{1}{a^{2}}\right)=\frac{1}{\mu} \exp (-\mu \operatorname{arccosh}(a))
$$

for any real $a \geq 1$ and any complex $\mu$ with a sufficiently large, positive real part; for the last equality here use, in particular, Lebedev (1972, Equation (9.8.3)). Hence, we obtain

$\mathrm{E}(\xi)=\mathcal{L}^{-1}\left(\frac{1}{\sqrt{2 z}} \exp (-\sqrt{2 z} \operatorname{arccosh}(a(x, \xi)))\right)(h)=\frac{1}{\sqrt{2 \pi h}} \exp \left(-\frac{\operatorname{arccosh}^{2}(a(x, \xi))}{2 h}\right)$, 
on using a standard Laplace transform from Doetsch (1971, Beispiel 8, pp. 50f.) for the last equality. To finally obtain the expression for the negative conditional moment of $A_{h}$, differentiate this equality with respect to $\xi$ and evaluate the result at $\xi=0$. The proof of Dufresne's integral representation for $m_{1}^{+}$is then complete.

\subsection{Characterizing solutions to Dufresne-type recurrences}

In this section we characterize functional recurrences of the same form as those of Dufresne recalled in Lemma 7.2. The precise result is as follows.

Proposition 7.2. Let $\left(F_{n}\right)_{n \in \mathbb{N}}$ be a sequence of differentiable functions on an open subset of the real line such that, for any integer $n \geq 1$, there are reals $a_{n}$ and $b_{n}$ for which

$$
F_{n+1}=a_{n} F_{n}^{\prime}+b_{n} F_{n} .
$$

Any such function is differentiable infinitely often and has the representation

$$
F_{n}=\sum_{k=1}^{n} A_{n, k} F_{1}^{(k-1)}
$$

in terms of $F_{1}$ and its derivatives, $F_{1}^{(\ell)}$. Here the reals $A_{n, k}$ are recursively defined by $A_{1,1}=1$, $A_{n+1,1}=b_{n} A_{n, 1}, A_{n+1, n+1}=a_{n} A_{n, n}$, and $A_{n+1, k}=b_{n} A_{n, k}+a_{n} A_{n, k-1}$ for any integer $k$ in $\{2, \ldots, n\}$.

Addendum 7.1. We have the combinatorial description

$$
A_{n+1, k+1}=\sum_{J_{k}} a_{J_{k}} b_{J_{k}^{\mathrm{c}}}
$$

for any nonnegative integers $n$ and $k \leq n$. Here the sum ranges over all $k$-tuples $J_{k}=$ $\left(j_{1}, \ldots, j_{k}\right)$ of strictly increasingly ordered integers $j_{\ell}$ in $\{1, \ldots, n\}$, and $J_{k}^{\mathrm{c}}=\left(\ell_{1}, \ldots, \ell_{n-k}\right)$ is the complement of $J_{k}$ in $\{1, \ldots, n\}$.

Proof of Proposition 7.2. The proof of Proposition 7.2 is by induction on $n$. The case $n=1$ holds by construction of $F_{1}$, so we assume the validity of the statement for any fixed, positive integer $n$. Using the recurrence for $F_{n+1}$, we thus obtain

$$
F_{n+1}=b_{n} A_{n, 1} F_{1}^{(0)}+\sum_{k=2}^{n}\left(b_{n} A_{n, k}+a_{n} A_{n, k-1}\right) F_{1}^{(k-1)}+a_{n} A_{n, n} F_{1}^{(n)},
$$

on collecting terms. Using the recurrence for $A_{n+1, k}$ stated in the proposition then completes the induction step and, thus, completes the proof.

Proof of Addendum 7.1. The description of the $A_{n+1, k+1}$ in Addendum 7.1 is proved by induction. To make explicit some pathological cases, for $n=0$ we have $k=0$, so the sum is over a single 0-tuple which, along with its complement, is empty. Hence, we have $A_{1,1}=a_{\varnothing} b_{\varnothing}=1$, since products over empty sets are equal to 1 by convention. Proceeding inductively, on applying the recurrences for $A_{n+1, k}$ of Proposition 7.2 we have $A_{n+1,1}=$ $b_{n} A_{n, 1}=b_{n} a_{\varnothing} b_{\{1, \ldots, n-1\}}=a_{\varnothing} b_{\{1, \ldots, n\}}$ and, similarly, $A_{n+1, n+1}=a_{\{1, \ldots, n\}} b_{\varnothing}$. If $k$ is in $\{2, \ldots, n\}$, then

$$
A_{n+1, k+1}=\sum_{J_{k}} a_{J_{k}} b_{J_{k}^{\mathrm{c}} \cup\{n\}}+\sum_{J_{k-1} \cup\{n\}} a_{J_{k-1} \cup\{n\}} b_{J_{k-1}^{\mathrm{c}}}
$$

completing the induction step and, thus, completing the proof of Addendum 7.1. 


\subsection{The moment decomposition theorem}

In this section we collect consequences of the results of Section 7.3, thus furnishing a first step in establishing Theorem 7.2. First we show how the weighted negative moments $m_{n}^{+}(h)=\mathrm{e}^{v^{2} h / 2} \mathrm{E}\left[\left(A^{(v)}(h)\right)^{-n}\right]$ of Section 7.2 are determined by the -1 th moment, $m_{1}^{+}$, and its derivatives. This is made precise in our following moment decomposition theorem.

Theorem 7.5. In the setting of Theorem 7.2, any negative moment function $m_{n}^{+}$is smooth on the positive real line and, in terms of $m_{1}^{+}$and its higher derivatives, $\left(m_{1}^{+}\right)^{(k)}$, has the representation

$$
m_{n}^{+}=\sum_{k=1}^{n} a_{n, k}\left(m_{1}^{+}\right)^{(k-1)} .
$$

In fact, the Proposition 7.2 coefficients $A_{n, k}$ specialize to the Theorem 7.2 coefficients $a_{n, k}$ when the Proposition 7.2 parameters $a_{n}$ and $b_{n}$ are specialized to the Theorem 7.2 parameters $\alpha_{n}$ and $\beta_{n}$, respectively. Given this observation, Theorem 7.5 follows immediately from Proposition 7.2, whose addendum now describes the combinatorial structure of the $a_{n, k}$.

Thus, a first explicit integral representation for $m_{n}^{+}$can be obtained by differentiation of Dufresne's integral for $m_{1}^{+}$, recalled in Lemma 7.1, as follows.

Theorem 7.6. For any positive integer $n$ and any positive real $h$, we have

$$
\left(m_{1}^{+}\right)^{(n)}(h)=\frac{2}{\sqrt{2 \pi}} \sum_{\left|I_{2}\right|=n} \sum_{k=0}^{n_{2}-1} c_{I_{2}, k} \frac{M_{2\left(n_{2}-k\right)+1}(h)}{h^{3 / 2+n_{1}+2 n_{2}-k}} .
$$

Here the first sum is over all pairs $I_{2}=\left(n_{1}, n_{2}\right)$ of nonnegative integers with sum $\left|I_{2}\right|=n_{1}+n_{2}$ equal to $n$.

To explain the additional concepts, the $c_{I_{2}, k}$ are constants given by

$$
c_{I_{2}, k}=(-1)^{n_{2}}\left(\begin{array}{c}
n \\
I_{2}
\end{array}\right)\left(\begin{array}{c}
3 \\
2
\end{array}\right)_{n_{2}}\left(\begin{array}{c}
n_{2} \\
k
\end{array}\right) \frac{\left(n_{2}-k\right) !}{\left(n_{2}-(k+1)\right) !}
$$

and $M_{a}$, for any real $a \geq 1$, is the function on the positive real line given by

$$
M_{a}(h)=\int_{0}^{\infty} y^{a} \exp \left(-\frac{y^{2}}{2 h}\right) \frac{\cosh ((v-1) y)}{\sinh (y)} \mathrm{d} y .
$$

A further study of the negative moments $m_{n}^{+}$of the integral of geometric Brownian motion $A^{(v)}$ which also yields workable numerics for them requires theta functions.

\subsection{Theta representation lemma}

This section is the second preliminary step in establishing Theorem 7.2, and identifies the reason for the occurrence of theta functions. We show how the hyperbolic quotient factor of the integrand of Dufresne's integral for $m_{1}^{+}$of Lemma 7.1 is the Laplace transform of a theta function if we admit finitely many correction terms. Adopting the terminology and results about theta functions presented in Section 3.1, this is made precise in our extension of a classical result to the following theta representation lemma. 
Lemma 7.3. For any real $\beta$, we have, on choosing the principal branch of the logarithm,

$$
\frac{\cosh ((2 \beta-1) \sqrt{z})}{\sqrt{z} \sinh (\sqrt{z})}=\int_{0}^{\infty} \mathrm{e}^{-z w} \vartheta(\beta \mid w) \mathrm{d} w+\sum_{\ell=0}^{\ell_{v}-1}\left(X_{a_{\ell}}-\mathcal{L}\left(\chi_{a_{\ell}}\right)\right)(z)
$$

for any complex number $z$ with positive real part, where $\ell_{v}$ is the smallest nonnegative integer $\ell$ such that $a_{\ell}=2 \ell+1-|2 \beta-1|$ is nonnegative.

The functions here are, for any complex $a$, given by

$$
\chi_{a}(t)=\frac{1}{\sqrt{\pi t}} \exp \left(-\frac{a^{2}}{4 t}\right) \quad \text { and } \quad X_{b}(z)=\frac{1}{\sqrt{z}} \mathrm{e}^{-b \sqrt{z}}
$$

for any positive real $t$ and, respectively, any $z$ in the complex plane with the nonpositive real line deleted. If $\operatorname{Re}(a)$ and $\operatorname{Re}\left(a^{2}\right)$ are nonnegative, they are related by the Laplace transform identity, $\mathcal{L}\left(\chi_{a}\right)(z)=\int_{0}^{\infty} \mathrm{e}^{-z t} \chi_{a}(t) \mathrm{d} t=X_{a}(z)$ for any complex $z$ with $\operatorname{Re}(z)>0$; see, for example, Doetsch (1971, Beispiel 8, pp. 50f.).

Proof of Lemma 7.3. Notice that $\sinh \left(z^{1 / 2}\right)=\mathrm{e}^{z^{1 / 2}}\left(1-\mathrm{e}^{-2 z^{1 / 2}}\right)$ in the denominator of the left-hand side of the identity of Lemma 7.3. With the square root mapping onto the right-hand half-plane, we thus develop the reciprocal of the second factor of this product into a geometric series, to obtain

$$
L(z):=\frac{\cosh (\alpha \sqrt{z})}{\sqrt{z} \sinh (\sqrt{z})}=\sum_{\ell=0}^{\infty} X_{2 \ell+1-\alpha}(z)+\sum_{\ell=0}^{\infty} X_{2 \ell+1+\alpha}(z),
$$

setting $\alpha=|2 \beta-1|$. As recalled above, the individual summands here are Laplace transforms of the corresponding functions $\chi_{a}$ if and only if $2 \ell+1-\alpha$ is nonnegative. By construction of $\ell_{\nu}$, we thus have

$$
L=\sum_{\ell=\ell_{v}}^{\infty} \mathcal{L}\left(\chi_{2 \ell+1-\alpha}\right)+\sum_{\ell=0}^{\infty} \mathcal{L}\left(\chi_{2 \ell+1+\alpha}\right)+\sum_{\ell=0}^{\ell_{v}-1} X_{a_{\ell}} .
$$

Since this series is absolutely convergent, interchanging the order of the Laplace transform integrals and the summations is justified, and the result is

$$
L=\mathcal{L}\left(\sum_{\ell=\ell_{v}}^{\infty} \chi_{2 \ell+1-\alpha}+\sum_{\ell=0}^{\infty} \chi_{2 \ell+1+\alpha}\right)+\sum_{\ell=0}^{\ell_{v}-1} X_{a_{\ell}} .
$$

In the first sum in brackets, successively shift the summation index by one, index the resulting sum by negative integers, and use the symmetry $\chi_{-a}=\chi_{a}$, to obtain the tautology

$$
L=\mathcal{L}\left(\vartheta\left(\frac{1}{2}(\alpha+1) \mid \cdot\right)\right)+\sum_{\ell=0}^{\ell_{v}-1}\left\{X_{a_{\ell}}-\mathcal{L}\left(\chi_{a_{\ell}}\right)\right\} .
$$

Since we have $\vartheta((\alpha+1) / 2 \mid w)=\vartheta(\beta \mid w)$, which for $2 \beta \leq 1$ follows from the Jacobi identity, the proof of Lemma 7.3 is thus complete. 


\subsection{Theta decomposition theorem}

This section is the third preliminary step in proving Theorem 7.2 and focuses on the computation of the higher derivatives of $m_{1}^{+}$, to which the computation of the weighted moments $m_{n}^{+}$has been reduced in the moment decomposition theorem of Section 7.4. Our discussion proceeds in three stages, from a structural point of view to a more computational one, with proofs of the results deferred to the end of the section. We use the functions $A_{a}, B_{a}, C_{a, m}$, and $\chi_{a}$ from Section 4.1. As a first step, the structure of the derivatives is described. This we do by identifying the exact contribution of the theta integrals $\Theta_{n}$ from Section 7.1, which are given by

$$
\Theta_{n}(h)=\int_{0}^{\infty} \vartheta\left(\frac{v}{2} \mid w\right) Q_{n}(h, w) \mathrm{d} w, \quad \text { where } Q_{n}(h, w)=\frac{(2 w)^{n-1}}{(2 h w+1)^{n+1 / 2}},
$$

for any integer $n \geq 1$. The precise result to be proved, our theta decomposition theorem, takes the following form.

Theorem 7.7. For any nonnegative integer $k$, the kth derivative, $\left(m_{1}^{+}\right)^{(k)}$, of $m_{1}^{+}$has the representation

$$
\left(m_{1}^{+}\right)^{(k)}=\Theta_{1}^{(k)}+\Delta^{(k)},
$$

with a correction term, $\Delta$, given, for any real $h>0$, by

$$
\Delta(h)=\sum_{\ell=0}^{\ell_{v}-1} \frac{2}{h} \mathrm{E}\left[W_{h} \mathrm{e}^{-a_{\ell} W_{h}}\right]-C_{a_{\ell}, 1}(h),
$$

where $\ell_{v}$ is the smallest nonnegative integer $\ell$ such that $a_{\ell}=2 \ell+1-|v-1|$ is nonnegative.

The constituents of the correction terms are identified as a second step. This identification runs in two directions, giving the nonprobabilistically defined functions of Section 4.1 a probabilistic interpretation, and vice versa. Our two results here are as follows.

Proposition 7.3. We have $(2 / h) \mathrm{E}\left[W_{h} \mathrm{e}^{-a W_{h}}\right]=\left(A_{1 / 2}-B_{a}\right)(h)$ for any real $h>0$.

Proposition 7.4. For any real $a \neq 0$ and any integer $n \geq 1$, we have

$$
C_{a, n}(B, h)=\frac{4}{|a|} \mathrm{E}\left[\mathbf{1}_{(1 / \sqrt{B}, \infty)}\left(W_{2 / a^{2}}\right) \frac{1}{W_{2 / a^{2}}^{2}} Q_{n}\left(h, \frac{1}{W_{2 / a^{2}}^{2}}\right)\right],
$$

where $B$ is any nonnegative (possibly infinite) real.

The last result can be checked by direct computation. Taken together, the last three results enable the following description of the derivatives of the Theorem 7.7 correction term $\Delta$.

Theorem 7.8. For any nonnegative integer $k$, the $k$ th derivative, $\Delta^{(k)}$, of the correction term function $\Delta$ is given as the sum of $k$ th derivatives by

$$
\Delta^{(k)}=\sum_{\ell=0}^{\ell_{v}-1}\left(A_{1 / 2}\right)^{(k)}-\left(B_{a_{\ell}}\right)^{(k)}-\left(C_{a_{\ell}, 1}\right)^{(k)},
$$

where $\ell_{v}$ is the smallest nonnegative integer $\ell$ such that $a_{\ell}=2 \ell+1-|v-1|$ is nonnegative. 
The point of these results is that they reduce the computation of derivatives of $m_{1}^{+}$to the computation of those of four explicitly given functions, which issue is to be addressed in the next sections. We now turn to proving this section's results, beginning with a preliminary lemma.

Lemma 7.4. All theta integrals $\Theta_{n}$ are well defined.

Proof. Let $f_{n}=\vartheta(v / 2 \mid \cdot) Q_{n}$ be the integrand of any $\Theta_{n}$. Majorize the theta function factor of $f_{n}$ by the appropriate majorizing function of Proposition 3.2. Then multiply the latter by $Q_{n}$ to obtain the majorizing function, $g_{n}$, of $f_{n}$. Integrability of $g_{n}$ on the positive real line is clear for $n \geq 2$ and is seen for $n=1$ on partial integration. The proof of Lemma 7.4 is thus complete.

Proofs of Theorem 7.7 and Proposition 7.3. The proof of Theorem 7.7 reduces to the case $k=0$, which we show while proving Proposition 7.3. First apply Lemma 7.3 with $\beta=v / 2$ and $z=y^{2}$ to the Lemma 7.1 integral for $m_{1}^{+}$, obtaining the representation

$$
m_{1}^{+}(h)=M_{1}(h)+\sum_{\ell=0}^{\ell_{v}-1}\left(M_{2, \ell}-M_{3, \ell}\right)(h)
$$

a sum of double integrals which we now define and identify. First, we have

$$
\begin{aligned}
M_{1}(h) & =\int_{0}^{\infty} \frac{2 y^{2}}{\sqrt{2 \pi h^{3}}} \exp \left(-\frac{y^{2}}{2 h}\right) \int_{0}^{\infty} \mathrm{e}^{-y^{2} w} \vartheta\left(\frac{v}{2} \mid w\right) \mathrm{d} w \mathrm{~d} y \\
& =\int_{0}^{\infty} \vartheta\left(\frac{v}{2} \mid w\right) \frac{\mathrm{d} w}{(2 h w+1)^{3 / 2}}=\Theta_{1}(h) .
\end{aligned}
$$

In the first double integral apply Tonelli's theorem and interchange the order of integration, noting that the integrands here are positive functions. The resulting inner integral is computed using $\int_{(0, \infty)} y^{2} \mathrm{e}^{-c y^{2}} \mathrm{~d} y=\left(\pi^{1 / 2} / 4\right) c^{-3 / 2}$ for any $c=w+1 /(2 h)$, whence the second equality is obtained. This last integral is equal to $\Theta_{1}(h)$ by definition, so all the integrals are finite a posteriori, by Lemma 7.4. Analogously, the identity

$$
M_{3, \ell}(h)=\int_{0}^{\infty} \frac{2 y^{2}}{\sqrt{2 \pi h^{3}}} \exp \left(-\frac{y^{2}}{2 h}\right) \int_{0}^{\infty} \mathrm{e}^{-y^{2} w} \chi_{a_{\ell}}(w) \mathrm{d} w \mathrm{~d} y=C_{a_{\ell}, 1}(h)
$$

follows by exchanging the theta function in the above for $\chi_{a}$.

For $M_{2, \ell}$ we have two identities:

$$
\begin{aligned}
& M_{2, \ell}(h)=\frac{2}{h} \int_{0}^{\infty} \frac{y^{2}}{\sqrt{2 \pi h}} \exp \left(-\frac{y^{2}}{2 h}\right) X_{a_{\ell}}\left(y^{2}\right) \mathrm{d} y=\frac{2}{h} \mathrm{E}\left[W_{h} \mathrm{e}^{-a_{\ell} W_{h}}\right], \\
& M_{2, \ell}(h)=\frac{2 \sqrt{2 h}}{\sqrt{2 \pi h^{3}}} \exp \left(\frac{a_{\ell}^{2} h}{2}\right) \int_{\left(a_{\ell} / 2\right) \sqrt{2 h}}^{\infty}\left(\sqrt{2 h} x-a_{\ell} h\right) \mathrm{e}^{-x^{2}} \mathrm{~d} x=\left(A_{1 / 2}-B_{a_{\ell}}\right)(h) .
\end{aligned}
$$

The first of these is a tautology, recalling that $X_{a}\left(y^{2}\right)=y^{-1} \mathrm{e}^{-a y}$ from Section 7.5. To obtain the second, first change variable according to $x=(2 h)^{-1 / 2}\left(y+a_{\ell} h\right)$ in the defining integral and then integrate the result. This completes the proofs of Theorem 7.7 and Proposition 7.3. 


\subsection{Derivatives}

Working in the setting of Section 7.6, in this section we relate the derivatives of the functions $\Theta_{n}, A_{a}, B_{a}$, and $C_{a, m}$ in Theorems 7.7 and 7.8 to these functions themselves. Recalling the Pochhammer symbols $(\lambda)_{k}$ of any complex $\lambda$, from Section 4 , the precise results here are as follows.

Lemma 7.5. We have $\left(A_{a}\right)^{(k)}=(-1)^{k}(a)_{k} A_{a+k}$.

Lemma 7.6. We have $\left(B_{a}\right)^{(k)}=b^{k} B_{a}-b \sum_{\ell=0}^{k-1} b^{k-1-\ell}\left(A_{1 / 2}\right)^{(k)}$, setting $b=a^{2} / 2$.

While the result of Lemma 7.5 is clear, the differentiation rule of Lemma 7.6 follows inductively from the differentiation rule $B_{a}^{\prime}=b B_{a}-b A_{1 / 2}$. Combined with the representation of Proposition 4.1 for the functions $G_{n}$ in terms of the weighted complementary error function, WErfc, Lemma 7.6 implies the following formula for the $k$ th derivatives of $B_{a}$, for negative real $a$.

Lemma 7.7. If $a<0$ then $B_{a}^{(k)}(h)=(-1)^{k}\left(\frac{1}{2}\right)_{k}|a| b^{k} G_{k}(b h)+2 a b^{k} \mathrm{e}^{b h}$, setting $b=a^{2} / 2$.

Indeed, returning to definitions in the formula of Lemma 7.6 for negative $a$, the proof of Lemma 7.7 is reduced to showing that

$$
a b^{k} \operatorname{WErfc}(-\sqrt{b h})=2 a b^{k} \mathrm{e}^{b h}+|a| b^{k} \operatorname{WErfc}(\sqrt{b h}),
$$

which follows from $\operatorname{Erfc}(-z)=2-\operatorname{Erfc}(z)$.

The derivatives of the remaining two functions are respectively given by the following two results.

Lemma 7.8. We have $C_{a, 1}(B, \cdot)^{(k)}=(-1)^{k}\left(\frac{3}{2}\right)_{k} C_{a, k+1}(B, \cdot)$.

Lemma 7.9. We have $\Theta_{1}^{(k)}=(-1)^{k}\left(\frac{3}{2}\right)_{k} \Theta_{k+1}$.

These two differentiation rules follow inductively from $\partial Q_{n} / \partial h=-\left(n+\frac{1}{2}\right) Q_{n+1}$ if differentiation of $\Theta_{1}$ and $C_{a, 1}(B, \cdot)$ to any order is effected by differentiating under the defining integral signs. To justify this way of taking derivatives it is sufficient to show that the integrands of any $\Theta_{n}(h)$ and $C_{a, n}(B, h)$ lie below integrable functions independent of $h$ if $h$ ranges over any bounded subinterval $[b, c]$ of the positive reals. In fact, the integrands of $\Theta_{n}(b)$ and $C_{a, n}(B, b)$ can be checked to do the job here. Integrability is clear for the latter, while it has been established for the former in proving Lemma 7.4. This proves Lemma 7.8 and Lemma 7.9.

\subsection{Proof of Theorem 7.2}

Summarizing the development up to now, in this section we give a proof of the Theorem 7.2 formula for the $n$th moment of $Y_{h, h}=h / A^{(v)}(h)$, for any positive integer $n$. As a first step, we apply Theorem 7.5 to obtain the representation

$$
\exp \left(\frac{\nu^{2} h}{2}\right) \mathrm{E}\left[Y_{h, h}^{n}\right]=h^{n} m_{n}^{+}(h)=\sum_{k=1}^{n} a_{n, k} h^{n}\left(m_{1}^{+}\right)^{(k-1)}(h),
$$

whose first identity is a tautology from Section 7.2. As a second step, we account for the derivatives of $m_{1}^{+}$by using the Theorem 7.7 theta integral representation, to obtain

$$
\exp \left(\frac{\nu^{2} h}{2}\right) \mathrm{E}\left[Y_{h, h}^{n}\right]=\sum_{k=1}^{n} a_{n, k} h^{n}\left(\Theta_{1}^{(k-1)}+\Delta^{(k-1)}\right)(h) .
$$


Using Lemma 7.9, we have $\Theta_{1}^{(k-1)}=(-1)^{k-1}\left(\frac{3}{2}\right)_{k-1} \Theta_{k}$. Here, using $\left(\frac{3}{2}\right)_{k-1}=2\left(\frac{1}{2}\right)_{k}$ gives

$$
\exp \left(\frac{v^{2} h}{2}\right) \mathrm{E}\left[Y_{h, h}^{n}\right]=\sum_{k=1}^{n} a_{n, k}^{*}\left(2 h^{k} \Theta_{k}(h)+\left(c_{k} \Delta^{(k-1)}\right)(h)\right) .
$$

Now introduce $c_{k}(h)=(-1)^{k-1} h^{k} /\left(\frac{1}{2}\right)_{k}$ as temporary notation, and note that

$$
a_{n, k}^{*}=h^{n} a_{n, k} / c_{k}(h) .
$$

Applying Theorem 7.8, the $k$ th weighted correction term derivative can be written as

$$
\left(c_{k} \Delta^{(k-1)}\right)(h)=\sum_{\ell-0}^{\ell_{v}-1} \Delta_{k}^{*}\left(a_{\ell}, h\right)
$$

where

$$
\Delta_{k}^{*}(a, h)=c_{k}(h)\left(\left(A_{1 / 2}\right)^{(k-1)}-\left(B_{a}\right)^{(k-1)}-\left(C_{a, 1}\right)^{(k-1)}\right)(h) .
$$

Recalling that by construction the $a_{\ell}$ are negative reals if $\ell<\ell_{v}$, the problem thus reduces to computing the derivative summands of any of these last functions when $a<0$. Using Lemma 7.5, we have

$$
c_{k}(h)\left(A_{1 / 2}\right)^{(k-1)}(h)=\frac{1}{k-\frac{1}{2}} A_{-1 / 2}(h) .
$$

Applying Lemma 7.7, since $a$ is negative, yields

$$
c_{k}(h)\left(B_{a}\right)^{(k-1)}(h)=2 a h \frac{(-h b)^{k-1}}{\left(\frac{1}{2}\right)_{k}} \mathrm{e}^{h b}+\frac{|a| h}{k-\frac{1}{2}}(h b)^{k-1} G_{k-1}(h b),
$$

setting $b=b_{a}=a^{2} / 2$. To compute the last derivative, successively apply Lemma 7.8 and Proposition 4.2 with $a<0$ and $B=\infty$, to obtain

$$
c_{k}(h)\left(C_{a, 1}\right)^{(k-1)}(h)=-2 h^{k} C_{a, k}(h)=-(2 /|a|)(h b)^{k} G_{k}(h b) .
$$

Rewriting the identities in terms of $G_{m}^{*}(x)=x^{m} G_{m}(x)$ completes the proof of Theorem 7.2.

\subsection{Basic setting for computing theta integrals}

Now we focus on proving the series for theta integrals $\Theta_{n}$ presented in Theorems 7.3 and 7.4. As a first step, in this section we define a basic framework for doing so. For any real $h>0$ and for any $B$ in $[0, \infty]$, we break the defining integrals of $\Theta_{n}$ at $B$, to obtain the decomposition

$$
\Theta_{n}(h)=F_{B}(h)+I_{B}(h),
$$

whose finite part, $F_{B}(h)$, and whose improper part, $I_{B}(h)$, are respectively given by

$$
F_{B}(h)=\int_{0}^{B} \vartheta\left(\frac{v}{2} \mid w\right) Q_{n}(h, w) \mathrm{d} w \quad \text { and } \quad I_{B}(h)=\int_{B}^{\infty} \vartheta\left(\frac{v}{2} \mid w\right) Q_{n}(h, w) \mathrm{d} w,
$$

where $Q_{n}(h, w)=(2 w)^{n-1} /(2 w h+1)^{n+1 / 2}$ from Section 4.1. Recalling from Section 3.1 the two series representations for $\vartheta(\beta \mid w)$ and their different convergence characteristics, the 
idea is to choose $B$ to be smaller than 1 . Since the defining series for $\vartheta(\beta \mid w)$ has excellent convergence properties for small values of $w$, we use it in the finite part, $F_{B}$, to obtain

$$
F_{B}(h)=\int_{0}^{B} \sum_{m \in \mathbb{Z}} \chi_{2 \gamma_{m}}(w) Q_{n}(h, w) \mathrm{d} w,
$$

where $\chi_{a}(w)=(\pi w)^{-1 / 2} \exp \left(-(a / 2)^{2} / w\right)$ from Section 3.1 and we define $\gamma_{m}=v / 2+m$. Using the functional equation of the theta function, the resulting Jacobi transform, on the other hand, has excellent convergence properties for large values of $w$. We thus use this series to represent the theta function factor in the improper part, $I_{B}$, to obtain

$$
I_{B}(h)=\int_{B}^{\infty} \sum_{m=0}^{\infty} \varepsilon_{m} \cos (\pi m v) \mathrm{e}^{-\delta_{m}^{2} w} Q_{n}(h, w) \mathrm{d} w,
$$

where $\delta_{m}=\pi m$ for all nonnegative integers $m$ and the $\varepsilon_{m}$ are as in Theorem 7.3.

\subsection{Proof of the series for theta integrals}

In this section we prove Theorems 7.3 and 7.4 together. The first of these summarizes the development up to now. From Section 7.9 we have the representation $\Theta_{n}=F_{B}+I_{B}$ for any theta integral as the sum of its finite-part function, $F_{B}$, and its improper-part function, $I_{B}$. Assume for a moment that term-by-term integration of these series is justified. Recalling the defining integrals of the functions $C_{a, m}$ and $D_{a, m}$ from Section 4.1, we then have, almost tautologically, the series representations

$$
\begin{aligned}
F_{B}(h) & =\sum_{m \in \mathbb{Z}} C_{2 \gamma_{m}, n}(B, h), \\
I_{B}(h) & =\sum_{m=0}^{\infty} \varepsilon_{m} \cos (\pi m \nu) D_{\delta_{m}, n}(B, h),
\end{aligned}
$$

with $2 \gamma_{m}=\nu+2 m$ and $\delta_{m}=\pi m$ and with $\varepsilon_{0}=1$ and $\varepsilon_{m}=2$ for $m \geq 1$, as required for Theorem 7.3. It thus remains to prove two things: to justify term-by-term integration and to show absolute convergence of the resulting series. As an application of Lebesgue dominated convergence, to do so it is sufficient to construct integrable majorizing functions for the absolute values of the integrands of $F_{B}(h)$ and $I_{B}(h)$. Such majorizing functions were constructed in proving Lemma 7.4, so the proof of Theorem 7.3 is complete.

To prove Theorem 7.4, notice that the Section 7.9 decomposition of $\Theta_{n}$ implies that $\Theta_{n}=F_{\infty}$ and $\Theta_{n}=I_{0}$. Using Theorem 7.3, the proof of Theorem 7.4 thus reduces to showing that

$$
\sqrt{2 \pi h^{-1}} h^{n} C_{a, n}(\infty, h)=\Psi\left(1, \frac{3}{2}-n ; b_{a} h\right) \quad \text { and } \quad 2 h^{n} D_{a, n}(0, h)=\Gamma(n) \Psi\left(n, \frac{1}{2} ; b_{a} / h\right)
$$

for any real $h>0$ and $a$ and any integer $n \geq 1$, setting $b_{a}=a^{2} / 2$. This follows from reduction to the classical integral representation $\Gamma(a) \Psi(a, c ; z)=\int_{0}^{\infty} \mathrm{e}^{-z x} x^{a-1}(1+x)^{c-a-1} \mathrm{~d} x$, valid if $a$ and $z$ both have positive real parts; see Lebedev (1972, Equation (9.11.6)). We exemplify this by establishing the first identity in the preceding display. To do so, in the defining integral of $C_{a, n}(\infty, h)$ in Section 4.1, change variable according to $w=1 /(2 h x)$ and then extract the reciprocal of $(1 / x)^{n+1 / 2}$ from the resulting denominator, to obtain

$$
C_{a, n}(\infty, h)=\frac{1}{\sqrt{2 \pi}} \frac{h^{3 / 2-1}}{h^{n}} \int_{0}^{\infty} \exp \left(-2 h\left(\frac{a}{2}\right)^{2} x\right)(x+1)^{-(n+1 / 2)} \mathrm{d} x .
$$


On comparison, the integral factor here is equal to $\Psi(a, c ; z)$ with $a=1, c=3 / 2-n$, and $z=b_{a} h$, as desired. The proof of Theorem 7.4 is thus also complete.

\section{Computational examples}

In this section we demonstrate our results by applying our ladder height Laguerre reduction series in the Asian option valuation framework developed in Section 6. In the setting of Section 6.1, we address the valuation of Asian options on a non-dividend-paying stock that have been issued today at time $t_{0}=0$ at the money. Assuming an interest rate of $\varpi=r=0.09$, the two dimensionless magnitudes for normalized valuation, $v$ and $h$, depend on the time to maturity, $T$, and the volatility, $\sigma$.

In all computations we have used the PARI/GP computer algebra system (version 2.1.0) of C. Batut, K. Belabas, D. Bernardi, H. Cohen, and M. Olivier (see http://pari.math.u-bordeaux.fr/) on an HP Visualize 200 Workstation.

\subsection{Principal remarks on normalized valuation}

The point of working with normalized prices $C^{(v)}$ is that the computation of the value, $C_{t}$, of any Asian option at time $t$ can be reduced to this dimensionless function of three dimensionless variables by way of the Section 6.1 factorization

$$
C_{t}=S_{t} \operatorname{norm}_{\sigma, t} C^{(v)}(h), \quad \text { where norm } \sigma, t=\frac{\mathrm{e}^{-r(T-t)}}{(\sigma / 2)^{2}\left(T-t_{0}\right)} .
$$

The cost of this simplification, however, is that normalized prices have to be calculated to greater precision for this reduction to be useful; see Schröder (2005c, Section 7.2) for more details. Our results now allow the computation of normalized prices to, in principle, any precision, and thus enable a benchmark valuation of Asian options in the mathematically strict sense. We demonstrate this point of view in the next three sections.

\subsection{Computing theta integrals and negative moments}

In this section we illustrate the theta integrals and negative moments which arise in valuing Asian options, by reference to results of Schröder (2005c, Section 9.1) in situations with $h=$ 0.0225 and $v=1$, values corresponding to $\sigma=30 \%$ and $T=1 \mathrm{yr}$.

Inspection of the Section 7.1 formulae for theta integrals suggests that they grow polynomially in the reciprocal of $h$. Thus, we focus on the weighted theta integrals $h^{n} \Theta_{n}(h)$, recorded in Table 1. They were computed using the Theorem 7.3 series with $B=0.3$, a value obtained using the estimates of Proposition 7.1. We have checked our results using the series of Theorem 7.4. We look at the numerical properties using the partial sum decomposition of $h^{n} \Theta_{n}(h)$ for Proposition 7.1 that defines the error terms $\rho_{k, M}=h^{n} R_{k, M}(B, h)$. In Table 1

TABLE 1: Growth of the weighted theta integrals $h^{n} \Theta_{n}(h)$.

\begin{tabular}{rccccc}
\hline$n$ & $M_{1,30}$ & $M_{2,30}$ & $M_{1,30}^{\text {est. }}$ & $M_{2,30}^{\text {est. }}$ & $h^{n} \Theta_{n}(h)$ \\
\hline 1 & 4 & 4 & 5 & 4 & 0.9962791864 \\
5 & 4 & 4 & 5 & 4 & 0.4063492063 \\
11 & 2 & 2 & 5 & 4 & 0.2702601836 \\
15 & 1 & 1 & 5 & 4 & 0.2307372767 \\
19 & 0 & 1 & 5 & 4 & 0.2046562496 \\
\hline
\end{tabular}


TABLE 2: Growth of the weighted negative moments $h^{n} m_{n}(h)$.

\begin{tabular}{rc}
\hline$n$ & $h^{n} m_{n}(h)$ \\
\hline 1 & 0.9851338557 \\
3 & 1.0449929081 \\
5 & 1.2437716541 \\
7 & 1.6544547393 \\
9 & 2.4502617752 \\
11 & 4.0257415110 \\
13 & 7.3124121451 \\
15 & 14.6363399787 \\
17 & 32.1813372115 \\
19 & 77.4965069820 \\
\hline
\end{tabular}

TABLE 3: Break-off values $n_{k}$ for replicating $k$ decimal digits of $C^{(v)}$, with $\lambda=0.65$.

\begin{tabular}{cccrrrc}
\hline$\sigma(\%)$ & $(v, h)$ & $C^{(v)}$ & $\alpha$ & $n_{5}$ & $n_{10}$ & $D_{150}$ \\
\hline 20 & $(3.5,0.01)$ & 0.000741559987883 & 45 & 6 & 14 & 37 \\
30 & $(1,0.0225)$ & 0.002173545046250 & 15 & 6 & 15 & 28 \\
40 & $(0.125,0.04)$ & 0.004781003283417 & 3 & 9 & 23 & 25 \\
50 & $(-0.28,0.0625)$ & 0.008909420452132 & 0 & 10 & 23 & 20 \\
\hline
\end{tabular}

we respectively denote by $M_{1,30}$ and $M_{2,30}$ the first indices $M$ for which we observe the first 30 decimal digits to be correct in the partial sums for $h^{n} F_{B}(h)$ and $h^{n} I_{B}(h)$. They decrease with increasing $n$. The $M_{k, 30}^{\text {est. }}, k=1,2$, on the other hand, are the lowest numbers of terms necessary to achieve this '30D' precision in the series predicted using the Proposition 7.1 estimates for the error terms $\rho_{k, M}$. They are constant: the estimates become independent of $n$ on multiplication by $h^{n}$. Using a crude implementation, computing the first 20 theta integrals for Table 1 required 2.53 seconds using 50D computing precison, 14.89 seconds using 100D computing precison, and 133.63 seconds using 200D computing precison. This seems in part due to PARI/GP's inability to evaluate the incomplete gamma functions particularly rapidly. However, precomputing the values of $G_{k}$ should speed up computation, in particular when $n$ is large and when higher computing precisions is used.

Using these weighted theta integrals, weighted negative moments of $A^{(v)}$ have been computed using Theorem 7.2, and recorded in Table 2. Their use in the series of Section 6 is illustrated in the next two sections.

\subsection{Computing with the Theorem 6.2 reduction series}

In this section we illustrate the Laguerre reduction series of Theorem 6.2. Benchmark values for the normalized prices $C^{(v)}(h)$ have been computed in Schröder (2002, Chapter V, Section 13) from a Hartman-Watson approach in the sense of Schröder (2003); see also Schröder (2005a, Section 8). To replicate these results, let $c=\lambda / q$ with $\lambda=0.65$, and keep variable the Laguerre parameters $\alpha$ that determine with which of the kinds of Section 2.1 Laguerre polynomials we work. Then determine the values $n_{k}$ at which to break off the summation of the Theorem 6.2 Laguerre reduction series in order to replicate the first $k$ decimal digits of our $C^{(v)}$-benchmarks. Our findings for these break-off values are recorded for four values of the volatility $\sigma$ in columns five and six of Table 3. 
TABLE 4: Estimated break-off values $\left(n_{5}^{\text {est. }}, n_{10}^{\text {est. }}\right)$ for correctness of $k$ decimal digits for $\sigma=30 \%$.

\begin{tabular}{rccc}
\hline \multirow{2}{*}{$\alpha$} & \multicolumn{3}{c}{$\lambda$} \\
\cline { 2 - 4 } & 0.6 & 0.65 & 0.7 \\
\hline 0 & $(19,27)$ & $(24,35)$ & $(31,45)$ \\
5 & $(14,25)$ & $(18,29)$ & $(24,37)$ \\
10 & $(9,25)$ & $(13,23)$ & $(17,30)$ \\
15 & $(6,24)$ & $(9,20)$ & $(12,24)$ \\
\hline
\end{tabular}

These results show that only a very limited number of series terms, in fact not more than 23 for the volatilities considered, are needed to obtain accuracy to at least 10 decimal places. In the last column of Table 3 we have recorded the number of stable decimal digits, $D_{150}$, observed on continuing the summation up to 150 terms. The results are satisfying in two particular respects. First, because of the small number of series terms and negative moments needed, using Laguerre reduction series gives a rapid way of computing normalized Asian option values to higher accuracies. Second, difficulties have been observed in computing them when volatilities or times to maturity are small in apparently all other approaches to valuing Asian options known today, including those of Linetsky (2004) and Schröder (2002). These difficulties are absent from our computations. As shown in the fourth column of Table 3, it is instrumental in this to vary the structural parameter $\alpha$ and, so, vary the Laguerre polynomial worked with.

For computation, the estimates for the approximation errors, $R_{N}$, of Theorem 6.2 are vital. This is because the parameters $\alpha$ and $\lambda$ strongly influence the convergence of the Laguerre reduction series. Table 4 illustrates how for $\sigma=30 \%$ we thus arrived at the choices $\alpha=15$ and $\lambda=0.65$ in Table 3 .

Finally, the parameter $\beta$ in the Laguerre reduction series of Theorem 6.2 affects the type of moments required. Choosing $L$ in the parametrization $\alpha-\beta-1=-L$ as a positive integer thus specifies the number of moments of $A^{(v)} / h$ in the series. From Theorem 7.1, these moments are available explicitly, so we try to replace the negative moments of $A^{(v)}$ by them. In our examples, it turns out that for accuracy to five decimal places we can thus dispense with moments of $Y_{h, h}$, although they are needed for higher accuracies. Principally, we have observed a value for $L$ that is maximal with respect to requiring the minimum number of such negative moments in the computation.

All these results are comparable to the better ones of Schröder (2005c), in which alternative Laguerre reduction approaches were developed.

\subsection{Computing with the Theorem 6.1 reduction series}

Working in the setting of Sections 8.2 and 8.3, in this section we address computation with the ladder height Laguerre reduction series of Theorem 6.1. With no error analysis available, we resorted to a search based on the results of Section 8.2. Our findings suggested the choice $\alpha=0$ and $\beta=0$, as in Dufresne (2000). The results for the parameter choices of Table 3 are presented in Table 5 .

In comparison with the results of Table 3 , here more series terms are required in order to replicate the $C^{(v)}$-benchmarks of Schröder (2002). Still, the computational effort required seems to be less than that required to establish the benchmarks in Schröder (2002), where computation times are of the order of CPU minutes. The latter results nevertheless seem to be far less time consuming and work intensive than the extremely hard-won results of Linetsky (2004), which are also highly experimental. 
TABLE 5: Break-off values $n_{k}$ for replicating $k$ decimal digits of $C^{(v)}$ with $\lambda=0.65$.

\begin{tabular}{ccccccc}
\hline$\sigma(\%)$ & $(v, h)$ & $C^{(v)}$ & $\alpha$ & $n_{5}$ & $n_{10}$ & $D_{150}$ \\
\hline 20 & $(3.5,0.01)$ & 0.000741559987883 & 0 & 47 & 61 & 42 \\
30 & $(1,0.0225)$ & 0.002173545046250 & 0 & 20 & 33 & 32 \\
40 & $(0.125,0.04)$ & 0.004781003283417 & 0 & 11 & 23 & 25 \\
50 & $(-0.28,0.0625)$ & 0.008909420452132 & 0 & 11 & 24 & 21 \\
\hline
\end{tabular}

\section{Epilogue}

In this paper we have focused on stochastic functionals $\mathrm{E}[\rho(Y)]$ of a given random variable $Y$, the integral of geometric Brownian motion at a fixed time. An explicit structure theory has been developed for them. In it we have established new interconnections between the integral of geometric Brownian motion and objects from other parts of mathematics such as theta functions, and have found these to be instrumental in actual computation. However, we have not been able to address the idea highlighted in Schröder (2006, Section 6.4) of using 'pure' ladder height density representations as a method of constructing stochastic functionals. This should become important in discrete-time problems, for instance enabling the construction of discretely sampled Asian option price functionals from observed 'plain-vanilla' option prices, a line of thought addressed in Schröder (2005b). In this sense the author would be more than gratified if Schröder (2006) and the present paper furnished starting points for further work connecting problems from finance, probability, and statistics.

\section{Acknowledgements}

The hospitality and support of the Mathematics Department of ETH, Zürich, in writing this paper, as well as partial support by the Deutsche Forschungsgemeinschaft via a project with Christian Hipp (Universität Karlsruhe (TH)), are gratefully acknowledged. Thanks go to the referee for comments and a careful reading of the text.

\section{References}

AliLI, L. (1995). Fonctionelles exponentielles et valeurs principales de mouvement Brownien. Doctoral Thesis, Université Paris VI.

Andrews, G. E., Askey, R. And Roy, R. (1999). Special Functions (Encyclopaedia Math. Appl. 71). Cambridge University Press.

CARR, P. AND SchröDER, M. (2004). Bessel processes, the integral of geometric Brownian motion, and Asian options. Theory Prob. Appl. 48, 400-425.

Comtet, A. And Monthus, C. (1996). Diffusion in a one-dimesional random medium and hyperbolic Brownian motion. J. Phys. A 29, 1331-1345.

DoetsCH, G. (1971). Handbuch der Laplace-Transformation, Band I. Birkhäuser, Basel.

Donati-Martin, C., Matsumoto, H. And Yor, M. (2000a). On striking identities about the exponential functionals of the Brownian bridge and Brownian motion. Periodica Math. Hung. 41, 103-119.

Donati-Martin, C., Matsumoto, H. and Yor, M. (2000b). On the positive and negative moments of the integral of geometric Brownian motion. Statist. Prob. Lett. 49, 45-52.

Donati-Martin, C., Matsumoto, H. and Yor, M. (2002). The law of geometric Brownian motion and its integral, revisited; application to conditional moments. In Mathematical Finance: Bachelier Congress 2000, eds H. Geman et al., Springer, Berlin, pp. 221-243.

DuFreSNE, D. (1989). Weak convergence of random growth processes with applications to insurance. Insurance Math. Econom. 8, 187-201.

Dufresne, D. (2000). Laguerre series for Asian and other options. Math. Finance 10, 407-428.

Dufresne, D. (2001). The integral of geometric Brownian motion. Adv. Appl. Prob. 33, 223-241. 
Dufresne, D. (2005). Bessel processes and Asian options. In Numerical Methods in Finance, eds M. Breton and H. Ben-Ameur, Springer, New York, pp. 35-58.

Erdélyi, A., Magnus, W., Oberhettinger, F. and Tricomi, F. G. (1953). Higher Transcendental Functions, Vol. 2. McGraw-Hill, New York.

Geman, H. And Yor, M. (1993). Bessel processes, Asian options, and perpetuities. Math. Finance 3, 349-375.

Karatzas, I. And Shreve, S. E. (1991). Brownian Motion and Stochastic Calculus, 2nd edn. Springer, New York.

Lebedev, N. N. (1972). Special Functions and Their Applications. Dover, New York.

LinETSKY, V. (2004). Spectral expansions for Asian (average price) options. Operat. Res. 52, 856-867.

McKean, H. P., JR. (1956). Elementary solutions of certain parabolic partial differential equations. Trans. Amer. Math. Soc. 82, 519-548.

Monthus, C. And Comtet, A. (1994). On the flux in a one-dimensional disordered system. J. Phys. I (France) 4, 635-653.

Mumford, D. (1983). Tata Lectures on Theta I (Progress Math. 28). Birkhäuser, Boston, MA.

Mumford, D. (1984). Tata Lectures on Theta II (Progress Math. 43). Birkhäuser, Boston, MA.

Mumford, D. (1991). Tata Lectures on Theta III (Progress Math. 97). Birkhäuser, Boston, MA.

Press, W. H., Teukolsky, S. A., Vetterling, W. T. and Flannery, B. P. (1992). Numerical Recipes in C, 2 nd edn. Cambridge University Press.

Rogers, L. C. G. And Shi, Z. (1995). The value of an Asian option. J. Appl. Prob. 32, 1077-1088.

Sansone, G. (1991). Orthogonal Functions. Dover, New York.

SCHRÖDER, M. (2002). Mathematical ramifications of option valuation: the case of the Asian option. Habilitationsschrift, Universität Mannheim.

Schröder, M. (2003). On the integral of geometric Brownian motion. Adv. Appl. Prob. 35, 159-183.

SCHRÖDER, M. (2005a). A constructive Hartman-Watson approach to stochastic functionals, with applications to finance. To appear in Theory Prob. Appl.

SCHRÖDER, M. (2005b). Continuous-time methods in the study of discretely sampled functionals of Lévy processes. I. The positive process case. To appear in Adv. Appl. Prob.

SCHRÖDER, M. (2005c). Laguerre series in contingent claim valuation, with applications to Asian options. Math. Finance 15, 491-531.

SchröDER, M. (2006). On ladder height densities and Laguerre series in the study of stochastic functionals. I. Basic methods and results. Adv. Appl. Prob. 38, 969-994.

VECER, J. (2001). A new PDE approach for pricing arithmetic average Asian options. J. Comput. Finance 4, $105-113$. VECER, J. (2002). Unified Asian pricing. Risk 15, 113-116.

Vecer, J. AND Xu, M. (2004). Pricing Asian options in a semimartingale model. Quant. Finance 4, 170-175.

Watson, G. N. (1944). A Treatise on the Theory of Bessel Functions, 2nd edn. Cambridge University Press.

Yor, M. (1992a). On some exponential functionals of Brownian motion. Adv. Appl. Prob. 24, 509-531.

Yor, M. (1992b). Some Aspects of Brownian Motion. Part I. Birkhäuser, Basel.

Yor, M. (ed.) (1997). Exponential Functionals and Principal Values Related to Brownian Motion. Revista Mathemática Iberoamericana, Madrid.

Yor, M. (2001). Exponential Functionals of Brownian Motion and Related Processes. Springer, Berlin. 